XXX.

\title{
Ueber syphilitische disseminirte, cerebrospinale Sklerose
}

\author{
nebst Bemerkungen über die secundäre Degeneration der \\ Fasern des vorderen Kleinhirnschenkels, des centralen Hauben- \\ bündels und der Schleifenschicht.
}

Von

Prof. W. v. Bechterew

in St. Petersburg.

(Hierzu Tafel XVII.)

Die Syphilis des Nervensystems ist in der letzten Zeit zum Gegenstande
zahlreicher Untersuchungen geworden und es hat sich dabei herausge-
stellt, dass die syphilitischen Affectionen die verschiedensten Gebiete des
Nervensystems befallen, hierbei nicht allein herdförmige, sondern auch
diffuse Verbreitung haben und zugleich sowohl acut wie auch chronisch
verlaufen können. Dank den Bestrebungen einer ganzen Reihe von
Autoren, unter welchen sich besonders die Namen von Fournier,
Marie, Rumpf, Kahler, Oppenheim, Erb u. A. bemerkbar machen,
ist es gelungen, einige von den syphilitischen Erkrankungsformen des
Nervensystems als selbstständige auszuscheiden und sie mehr oder we-
niger gut klinisch und pathologisch-anatomisch zu skizziren. Trotzdem
aber harrt auf diesem weiten Gebiet noch sehr viel Material einer wei-
teren Bearbeitung. Wie weit die Ansichten der Autoren über die syphi-
litischen Affectionen des Nervensystems noch auseinandergehen, beweist
unter Anderem der Umstand, dass sogar eine solche, allem Anschein
nach gut studirte Krankheitsform, wie die Tabes dorsalis noch Gegen-
stand der Controverse bildet, und bekanntlich von einigen Autoren als
eine Affection syphilitischen Charakters hingestellt wird. Für uns er-
scheint die Syphilis des Nervensystems als eine specifische, un-
mittelbar durch die luetische Infection bewirkte und nicht 
nur auf syphilitischem Boden sich entwickelnde Erkrankung des letzteren, welche in Anbetracht ihres pathologisch-anatomischen Wesens durch keinen anderen Process bedingt sein kann. Sogar bei einer solchen Einschränkung des Begriffes der Hirnsyphilis besitzen wir eine bedeutende Zahl einzelner Erkrankungsformen unzweifelhaft syphilitischer Natur.

U. A. unterscheidet Mari $\mathrm{e}^{*}$ ), dessen Erfahrungen in Bezug auf die Syphilis des Nervensystems wohl keinem Zweifel unterliegen kömnen, unter den acuten syphitischen Erkrankungen des Rückenmarks: eine acute syphilitische Myelitis, eine acute, ascendirende syphilitische Paralyse, die „meningeale Irritation" Lang's und die "secundäre syphilitische Analgesie" Fournier's; und unter den chronischen Formen: die chronische, einseitige, multiple, ccrebrospinale, syphilitische Affection(Lues cerebrospinalis Op p enheim's) und die syphilitische Spinalparalyse ron Erb. Trotz der vielleicht zu grossen Länge dieses Registers fehlen darin doch solche Formen, wie die Pseudotabes syphilitica, dje gummösen Geschwülste des Rückenmarks und die syphilitische Periostitis der Wirbelsäule. Auch im Gehirn stossen wir auf eine ganze Reihe von Erkrankungsformen von speciell syphilitischer Natur. Vou den gesicherteren Formen können hier erwähnt werden: die syphilitische Basilarmeningitis, die syphilitische Meningitis der Gehirnoberfläche, die syphilitische Arteriitis, die syphilitische Meningoencephalitis, die gummösen Geschwülste und die syphilitische Periostitis der Schädelknochen. Es ist jedoch zu bemerken, dass trotz der auffallenden Menge und Verschiedenheit der syphilitischen Affectionen des Nervensystems dieselben noch bei Weitem nicht als klinisch erforscht gelten können. Ganz im Gegentheil ist in dieser Hinsicht noch Vieles unaufgeklärt und im Grunde geuommen fehlen uns noch feste differentialdiagnostische Anhaltspunkte zur Unterscheidung der Syphilis des Nervensystems von analog Iocalisirten nichtsyphilitischen Affectionen. Mehr als für andere Formen ist es geglückt die syphilitische Spinalparalyse von Erb klinisch zu erforschen, und doch besitzen wir auch für diese Erkrankung, trotz einer ganzen Reihe von sehr eingeheaden in letzterer Zeit erschienenen Untersuchungen, streng genommen keine ganz bestimmten klinischen Symptome, welche uns die Möglichkeit gewährten, diese syphilitische Affection in jedem Falle von einer einfachen, circumscripten, gleich localisirten Myelitis zu unterscheiden.

Ohne Zweifel gebührt aber der syphilitischen Spinalparalyse nicht allein die Ehre der eingehenden Erforschung. In practischer Hinsicht besitzen andere Formen von Hirnsyphilis nicht geringe Bedeutung. Von

*) Marie, Semaine méd. No. 5. 1893. 
diesen soll eine weniger bekannte und in der Form von zerstreuten Herden sowohl im Rückenmark wie auch im Gehirn auftretende Erkrankung hier einer näheren Betrachtung unterworfen werden. Ein typisches Beispiel liefert folgender Fall.

Patient A., Officier, suchte den 17. Angust 1888 bei mir in Kasan ärztlichen Rath. Derselbe klagte über Schwäche in beiden Füssen und Schmerzen in verschiedenen Körpertheilen, welche letzteren sich den 1. August in den Seiten, im Rücken und theilweise auch in den Händen eingestellt hätten. Der Kranke erklärte ausserdem, dass es ihm schwer falle zu liegen, weil sich bei ihm hierbei ein unangenehmes Gefühl von Taubheit einstelle und es ihm vorkomme, als ob die Stelle dureh langes liegen gefühllos geworden sei. Diese Sehmerzen und Parästhesien hätten annähernd bis zum 7. August angehalten und seien anch in den Füssen aufgetreten. Es kam ihm vor, dass seine Füsse anschwellten, "als ob alles an ihnen herunterhänge." Ueberhaupt verspürt er nicht selten in den Füssen die Empfindung, ,als ob er gegen 30 Werst gegangen wäre." Die objective Untersuchung ergab folgendes: Patient von hohem Wuchs, mässig stark gebaut, das subcutane Fettpolster schwach entwickelt; keine auffallenden Störungen an den Brust- und Bauchorganen; die Wirbelsärle nicht yon der Norm abweichend. Am unteren Theil des Rückgrats, verursacht. tiefe Percussion einigen Schmerz. Dex Gang des Kranken zeigt eine eigenthiünliche Störung: es erscheint ihm gleichsam schwer, die Füsse zu strecken, weshalb er sie beim Gehen in den Kniegelenken leicht gebeugt lässt. Beim Stehen mit geschlossenen Augen sieht man ein schwaches Schwanken. Die Kniereflexe sind an beiden Extremitäten mässig gesteigert und bei passiver Beugung der Fussgelenke zeigt sich ein kurzdauernder klonischer Krampf (schwaches Fussphänomen). Der Sohlenreflex wird leicht erhalten, ohne jedoch eine besondere Steigerung aufzuweisen. Die oberen Extremitäten waren nicht afficirt und die Sehnenreflexe erschienen hier nicht erhöht. Die Sensibilität weist keine objectiv bemerkbare Abweichung weder an den Füssen noch an anderen Köpertheilen auf. Ausserdem ist zu vermerken, dass vor ca.10 Tagen sich bei dem Kranken Verstopfung eingestellt hatte und dass der Harnabsatz ihm in den letzten drei Tagen einige Schwierigkeiten macht.

Aus der Anamnese entnehmen wir ferner, dass der Kranke offenbar weder hereditär belastet noch besonderen Excessen und Erkältungen ausgesetzt gewesen war, anch an keiner acuten Infeetionskrankheit gelitten hatte. Vor etwa einem Jahre an Syphilis erkrankt, welche vom Professor für Haut- und venerische Krankheiten A. Gay rechtzeitig diagnosticirt wurde, hatte der Kranke sich dennoch keiner regelrechten antisyphilitischen Behandlung unterworfen. In Anbetracht einer solchen Anamnese verordnete ich ihm Einreibungen von grauer Quecksilbersalbe zu einer halben Drachme täglich, innerlich Jodnatrium in grossen Dosen und zur Abführung Pillen aus Extr. Rhei und Extr. Aloës aquos. Aus̃serdem musste der Patient täglich warme Salzbäder nehmen und seine Wirbelsäule wurde in absteigender Richtung galvanisirt. Auf die schmerzhafte Stelle des Rückgrats wurde ein Blasenpflaster applicirt und dasselbe in 
den nächsten Tagen wiederholt. Unter dem Einfluss dieser Therapie wurde die erwähnte Empfindlichkeit der Wirbelsäule schon in einigen Tagen bedeutend schwächer und das subjective Befinden des Patienten überhaupt besser, obwohl die objectiven motorischen Störungen unverändert geblieben waren. Da nach meinem besten Dafürhalten die Affection des Nerrensystems von der Syphilis abhing, so überwies ich den Kranken zur weiteren Behandlung an Prof. A. Gay, welcher auch eine systematische antisyphilitische Cur mitQuecksilberinjectionen, innerlichem Gebrauch von Jodpräparaten und Anwendung von warmen Bädern durchführte. Unter dem Einfluss einer solchen Behandlung erfolgte eine unzweifelhafte Besserung in dem Zustande des Kranken. Er fühlte sich fester auf den Füssen, sein Gang war merklich besser, die Beschwerden beim Harnabsatz und die früheren, den Patienten beunruhigenden, subjectiven Empfindungen waren fast verschwunden. Doch war bei ihm ausser einiger Störung des Ganges, wie früher ein geringes Schwanken nach Augenschluss, deutliche Steigerung der Patellarreflexe und klonischer, wenn auch kurzdauernder Krampf bei der Beugung des Fussgelenkes an beiden Füssen noch immer vorhanden. Hit dem Resultat der Behandlung zufrieden, reiste er den 3. Oct. desselben Jahres (1888) aus Kasan nach seinem Heimatsorte Pensa, trotzdem ihm gerathen worden war die Behandlung weiter fortzusetzen. In seiner Heimath fühlte sich der Kranke die erste Hälfte des October hindurch vollkommen wohl, so dass er sogar den 16. October auf der Hochzeit von seinen Bekannten am Tanze sich ketheiligte, wobei er nach seiner Meinung sich wohl erkäl tet hätte. Trotzdem blieb sein Befinden bis zum 20. October gut, an welchem Tage er aber in einen fieberhaften Zustand gerieth, worauf am folgenden Tage, den 21. October, er eine solche bedeutende Schwäche in den Füssen verspürt habe, dass er sich nur mit Mühe vom Bett zum Lehnstuhl begeben konnte. Die Rede erwies sich als stark behindert, und es bestand eine Deviation der Augen, so dass alle Gegenstände sich zu bewegen schienen. Den 22. October entwickelte sich eine fast vollständige Lähmung in den Füssen, weshalb der Kranke den 25. October sich in das locale Hospital aufnehmenl iess, wo er bis 10. Mai verblieb und sein Zustand sich ein wenig gebessert habe. Gegen Mitte Mai traf er in Kasan mit folgendem Status ein: In der Stellung und in den Bewegungen der Augen keine Abweichung; keine Diplopie; einige Behinderung der Aussprache, wenn auch keine bedeutende; die Bewegungen der Hände frei, nur an den Fingern ein geringes Zittern; eine fast vollständige spastische, das Gehen fast ganz unmöglich machende Paraplegie. Bei der Untersuchung wurde eine äusserst starke Steigerung der Patellarsehnenreflexe constatirt und bei der Bengung der Fussgelenke stellte sich unverzüglich eine starke clonische Erschütterung der ganzen Unterextremität ein. In sitzender und liegender Stellung. hielt der Kranke die Füsse ausgestreckt und ihre passive Beugung ist im höchsten Grade erschwert. Ist jedoch der erste Widerstand allmälig überwunden, so vermag man die Füsse zu beugen ohne auf ferneren Widerstand zu stossen. Die Sensibilität der Füsse, gleichwie anderer Körpertheile weist keine auffallende Schwächung auf. Die Harnentleerung ist erschwert und es besteht zugleich Impotentia virilis, auch ist Obstipation vorhanden. Bei derPercussion 
der Wirbelsäule wird wieder einige Empfindlichkeit ihres unteren Abschnittes vorgefunden. Zu vermerken ist, dass der Kranke eine äusserst reizbare Schwäche zeigt, so dass er bei dem geringsten Anlass weint. Ausserdem waren bei ihm in der letzten Zeit apoplectiforme Anfälle, im Ganzen zwei, aufgetreten. Da der Kranke zu Hause weder Bäder haben, noch sich galvanisch behandeln lassen konnte, so trat er in das hiesige Militärhospital, wo ausser Bädern und der Galvanisation bei ihm noch eine Jod- und Quecksilberbehandlung (subcutane Injectionen) eingeleitet wurde. Da im Verlaufe eines ganzen Monats sein Befinden im Hospital nicht besser geworden war, so ersuchte er um Abfertigung auf Kronkosten nach den Schwefelbädern zu Sergiewsk. Im Herbst kehrte er jedoch ron den Bädern in einem noch schlimmeren Zustande zurück und fand wieder im Militärhospitale Aufnahme. Dieses Patienten wegen in das Hospital zur Consultation aufgefordert, fand ich bei ihm schon eine bedeutende Erschwerung der Aussprache von Wörtern und eine stark ausgesprochene reizbare Schwäche, in Folge deren er bei dem geringsten Anlass in ein unaufhaltsames Weinen ausbrach. Unzweifelhaft besass dieses Weinen den Charakter eines gewaltsamen, weil es sich fast anlasslos entwickelte und der Kranke unfähig war sich desselben zu enthalten. Wie früher bestanden die Erscheinungen einer spastischen Paraplegie, begleitet von Störungen der Blasen-, Darm- und Geschlechtsfunctionen, bei erhaltener Sensibilität. Als ich den Kranken später wiedersah, hatte sich sein Zustand trotz der im Hospital eingeleiteten Behandlung (Bäder, Galvanisation längs der Wirbelsäule, innerlich Jodkalium) gar nicht gebessert. Auf seinen Wunsch wurde der Kranke im Frühling 1890 wieder nach den Sergiewsk' schen Mineralbädorn geschickt, wo er sehr sorgfältig Bäder nahm. Hier im Bade schien anfänglich Alles sich zum Bessern wenden zu wollen; gegen den Schluss der Saison aber erfolgte eine schroffe Verschlimmerung. Der Kranke war äusserst schnell dermaassen schwach geworden, dass er sich kaum aus der Wanne zu entfernen vermochte. Wieder kehrte er vom Badeorte in einem schlimmeren Zustande zurück, als er hingereist war. Im Herbste 1890 in Kasan eingetroffen, wurde er wieder in's Militärhospital placirt und nun mit Jod behandelt. Es lag die Absicht vor, an ihm die Behandlung mittels Suspension zu versuchen, was aber aufgegeben werden werden musste, da sein Zustand anfing sich progressiv zu verschlimmern. Allmälig wurde er immer schwächer und es stellte sich bei ihm in der rechten Hand und in der rechten Gesichtshälfte Parese ein. Laut Bericht des Hospitalarztes K. Sokolow hatte sich anfangs October bei dem Kranken eine fast vollkommene Lähmung der rechten Hand ausgebildet, so dass er nicht mehr im Stande war, dieselbe zu erheben. Zugleich verstärkte sich die Sprachstörung dermaassen, dass er schwer zu verstehen war, anch konnte er die Zunge nicht herausstrecken. Eine Woche später stellte sich eine Lähmung der Kaumuskeln ein. Nicht allein, dass der Kranke unfähig war, die Speise zu zerkauen, er konnte sogar den Mund nicht öffnen (Trigeminuslähmung). Da beim geöffneten Munde zwischen den Zahnreihen nur ein. schmaler Spalt vorhanden war, so konnte er nur flüssige Nahrung za sich nehmen. Gegen Mitte October entwickelte sich bei ihm eine Selstörung mit dem Charakter einer linksseitigen 
Hemianopsie. Hierbei war weder eine Störung der Hautempfindlichkeit noch eine solche des Gehörs bei dem Patienten wahrzunehen. Das Bewusstsein blieb während der ganzen Zeit erhalten; es bestand nur eine äusserst reizbare Schwäche und eine auffallende Neigung zum Weinen. Am Morgen des 20. October 1890 verschied der Kranke, nachdem er kurz vor dem Tode in einen comatösen Zustand verfallen war.

Da bei der am nächsten Tage, den 21. Oetober, erfolgten Obduction an den inneren Organen keine irgend welche besondere Beachtung verdienenden Veränderungen vorgefunden worden, so will ich hier nur das Sectionsprocoll des von mir untersuchten centralen Nervensystems, welches höchst ausgesprochene und äusserst interessante Veränderungen aufwies, mittheilen.

Die harte Hirnhaut mit den Schädelknochen längs der Sagittalnaht verwachsen; an ihrer inneren Fläche eine dicke Auflagerung von hämorrhagischer Pachymeningitis; an der Gehirnoberfläche eine kaum merkliche Trübung der Pia längs den Gefässen. An der Gehirnbasis deutliche Piatrübung in der Gegend der Fossa Sylvij, dann in der Gegend der Varolsbrücke und des verlängerten Markes, an welcher Stelle, wie auch am oberen Rückenmarissabschnitte sich sogar ein nicht umfangreiches, fibrinöses Exsudat findet. Beide Vertebralarterien fast normal, die Basilaris aber bedeutend verdickt (bis zu 0,5 Ctm. im Durchmesser), hart anzufühlen und mit knotigen Auftreibungen und Unebenheiten in ihrem Verlauf, dabei auch S-förmig gebogen. Am unteren Theil der Varolsbrücke ist die Art. basilaris mit den weichen Hirnhöhlen so fest verwachsen, dass die Trennung den Gebrauch eines Skalpels erfordert. Am Durchschnitt durch die Basilaris erblickt man eine äusserst starke Verdickung ihrer Wandungen, besonders an den aufgetriebenen Stellen, welche eigentlich von örtlichen Verdickungen der Gefässwand herrühren. Die Gefässlichtung erscheint sogar an den aufgetriebenen Stellen sehr eng und in Folge eines wandständigen Thrombus hatte dieselbe an einer Stelle nicht einmal die Grösse eines Stecknadelkopfes. An der rechten, vorderen oberen Kleinhirnarterie sieht man in der Nähe ihrer Abgangsstelle von der Basilaris eine starke, kugelige Verdickung mit einem Diameter von ca. 0,5 Ctm. Ein Durchschnitt durch dieselbe lehrte, dass diese Verdickung ausschliesslich durch eine solche der Wände und nicht durch eine Erweiterung der Lichtung, welche normal war, verursacht war. An dem rechten, hinteren Verbindungszweige wurde gleichfalls eine ähnliche Verdickung vorgefunden; dieselbe war mit den weichen Hirnhöhlen und mit der Rindensubstanz der rechten Schläfenlappenspitze (Gyrus uncinatus) verwachsen.

Die Lumina beider Carotiden klaffen. Auch an den mittleren Hirnarterien und ihren Ferzweigungen finden sich Wandverdickungen und sklerotische Herde. An den vorderen Gehirnarterien, in der Nähe ihrer Ursprnngsstellen, sieht man ebenfalls Verdicknngen der Wände und Verwachsungen mit den angrenzenden Hirnhüllen und der Gehirnsubstanz. Im unteren Abschnitt der Varolsbrücke, an der Verwachsungsstelle der Basilararterie mit der Hirnsubstanz, bemerkt man nach der Entfernung der Hirnhüllen eine graue Verfärbung derHirnoberfläche. Ein durch diese Brüclenstelle geführter Schnitt lässt einen 
grossen, granen, sklerotischen Herd, welcher bis $0,5 \mathrm{~cm}$ in die Tiefe geht und eine Haemorrhagie in sich birgt, erkennen. An seiner Peripherie ist aber eine Gewebserweichung zugegen. In den Ventrikeln befand sich eine geringe Menge klarer cerebrospinaler Flüssigkeit; die hinteren Ventrikelhörner, besonders des linken Ventrikels, erschienen erweitert. In der linken Hemisphäre war der Plexus chorioideus mit der Oberfläche des Ammonshornes fest verwachsen, und auch hier war ein sklerotischerHerd vorhanden. Ferner wurde ein sklerotischer Herd von grauer Farbe und mit einem mehr als $0,5 \mathrm{~cm}$ betragenden Durchmesser an der Verwachsungsstelle des rechten, hinteren Verbindungszweiges mit der Schläfenlappenspitze, in der Rindensubstanz der letzteren, angetroffen. Dieser Herd hatte sich auch auf den hier verlaufenden, rechten Tractus opticus verbreitet. Der rechte Plexus chorioideus ist mit dem hinteren Theil des Sehhügels verwachsen, und auch hier findet sich ein graner, sklerotischer Herd. In dem linken Sehhügel befand sich eine ziemlich harte Geschwulst von rundlicher Form und von der Grösse einer Erbse, welche alle charalkteristischen Eigenthümlichkeiten eines Gumma aufwies. In der Nachbarschaft dieser Geschwulst wird eine den ganzen mittleren Theil des Hügels einnehmende Gewebserweichung constatirt. Im Rückenmark wurde an der vorderen Fläche des oberen Theiles der Cervicalanschwellung, dem Verlaufe der vorderen Arterie entsprechend, an einer circa 0,5 cm langen Strecke ein schmaler, grauer, sich über die Oberfläche des Markes hervorwölbender, ziemlich derber Herd bemerkt. Als derselbe durchschnitten wurde, erwies es sich, dass der noch offene Stamm der vorderen Arterie durch denselben geht. Das Blutgefäss ist vom straffen, mit der Rückenmarkssubstanz, von welcher ein Theil mit den Herd bildet, ein organisches Ganze repräsentirenden Gewebe umgeben. Ebenfalls in derCervicalanschwellung des Rückenmarks, etwas unterhalb des beschriebenen Herdes, findet sich rechts, an der Austrittsstelle der vorderen Wurzeln ein gleicher, aber halbsogrosser. Herd von demselben Charakter. Derselbe wölbt sich ebenfalls über die Rückenmarksoberfläche hervor, da die aus dem Rückenmark tretenden Wurzeln mit demselben fest verwachsen sind. Weiter sieht man an dem oberen Brustabschnitt, links, an der Austrittsstelle der vorderen Wurzeln einen grossen, $3 \mathrm{~cm}$ langen Herd. Die hier austretenden Nervenwurzeln und die weiche, mit der unter ihr befindlichen Marksubstanz verwachsene Hirnhaut bilden mit demselben ein gemeinschaftliches Gewebe von grauer Farbe. An der nächsten Umgebung dieses Herdes weist das Rückenmarksgewebe deutliche Kenuzeichen der Erweichung auf. Im mittleren Theil des Brustabschnittes des Rückenmarks, an der vorderen Rückenmarksspalte, sieht man ebenfalls einen derben, grauen, sich hervorwölbenden Herd, welcher die Grösse eines Hirsekornes nicht ganz erreicht. Etwas unterhalb desselben, an der linken Seite, zeigt sich gleichfalls ein länglicher, mehr als $1 \mathrm{~cm}$ langer, grauer, an der Austrittsstelle der vorderen. Wurzeln befindlicher Herd. Wie an anderen Stellen sind die Wurzeln mit dem darunter liegenden Gewebe verwachsen und bilden einen Bestandtheil des Herdes. Ferner wurde an der Uebergangsstelle des Brust- in den Lendenabschnitt des Rückenmarks, ebenfalls an der linken Seite, ein derber Herd ron der Grösse einer kleinen Erbse, diesmal aber innerhalb der 
vorderen Wurzeln, welche an dieser Stelle in eine gemeinsame feste Masse von grauer Farbe verwachsen erschienen, angetroffen. Fast in demselben Niveau des Rückenmarks fängt ein grösserer, länglicher Herd rechts, an der Austrittsstelle der vorderen Wurzeln an und zieht sich beinahe $4 \mathrm{~cm}$ in der Richtung nach unten, längs der Austrittsstelle der vorderen Wurzeln des unteren Brustabschnittes und der Lendenanschwellung hin. Ebenso wie an anderen Orten ist auch hier die weiche Hirnhaut und ein Theil der vorderen Wurzeln mit dem Herd in ein organisches Ganze verlöthet. Am Niveau dieses Herdes findet sich im Hirngewebe eine ansgebreitete Erweichung, weshalb das Gewebe beim Durchschneiden dieses Rückenmarkstheiles sogleich auseinanderfliesst. Ausser allen diesen soeben aufgezählten Herden wurden bei der mikroskopischen Untersuchung noch einige Herde geringeren Umfanges sowohl im Rückenmark wie im Gehim angetroffen. UnterAnderem befanden sich zwei kleine Herde an der Seitenfläche des verlängerten Markes und zwar einer von ihnen im linken Corpus restiforme, an der Uebergangsstelle desselben in das Kleinhirn. Ausserdem war ein Herd in der Haube des linken Hirnschenkels, unter dem hinteren Zweihügel, in der Gegend, wo das centrale Haubenbündel durch den oberen Kleinhirnschenkel hindurchtritt. Die Grösse jedes dieser Herde überstieg nicht die eines Hirsekornes. Bei der Untersuchung der Schnitte aus diesen Herden war es nicht schwer, sich davon zu überzeugen, dass dieselben aus straffem, grauem oder grauröthlichem, eine glatte Schnittfläche lieferndem Gewebe bestehen. Das verdichtete, narbige Gewebe des Herdes reicht gewöhnlich bis zu einer mehr oder weniger bedeutenden Tiefe in das Hirngewebe hinein, und schon bei geringen Vergrösserungen lässt sich dabei in ihrer Umgebung starke Gefässentwickelung erkennen. In der Nachbarschaft einiger Herde fand sich sogar Erweichung der umgebenden Gewebe an einer mehr oder weniger weiten Strecke vor. AIs eine allgemein charakteristische Eigenthämlichkeit aller Herde erscheint ausserdem der Umstand, dass sie alle an solchen Stellen placirt sind, wo dem Hirngewebe dieses oder jenes Gefäss anliegt, welches alsdann mit dem Hirngewebe fest verwächst, oder aber durch das Hirn selbst hindurchgeht und allseitig von straffem, gleichsam narbigen Bindegewebe umgeben erscheint.

Indem wir nun zur mikroskopischen Untersuchung übergehen, wollen wir vor Allem die Veränderungen der grossen Gefässstämme an der Gehirnbasis betrachten. Wie wir gesehen, boten die Basilararterie und ebenso eine der Kleinhirnarterien Auftreibungen auf Kosten einer übermässigen Verdickung der Gefässwandungen dar. Die aus solchen aufgetriebenen Stellen der Basilaris und Art. cerebelli stammenden Schnitte überzeugten uns, dass diese Auftreibungen durch eine Verdickung hauptsächlich der äusseren und inneren Gefässhaut, besonders der ersteren zu Stande kommen (Fig. 1 u. 2). Hierbei wurde in dem Gewebe der Gefässwandung starke Entwickelung von Granulationselementen angetroffen, in Folge dessen die mittlere Gefässhaut atrophisch erschien und stellenweise sogar die innere elastische, die Media von der Intima scheidende Membran zum Schwinden gebracht worden war (Fig. 1). Ausserdem wurden in einigen von diesen Gefässauftreibungen wandständige, theil- 
weise schon organisirte Pfropfen rorgefunden. Ferner verdienen Beachtung die stellenweise in den pathologisch veränderten Gefässwandungen selbst angetroffenen Hämorrhagien, welche aus den Vasa vasorum zu stammen schienen, da letztere im Allgemeinen ebenso verändert waren, wie die grossen Gefässstämme. Somit ist es klar, dass in den Gefässen eine Arteriitis rorliegt und dass dieselbe unzweifelhaft syphilitischen Ursprungs ist, wofür nicht allein ihr eigenthümliches Gepräge und ihre Multiplicität, sondern auch die anderen, oben angeführten Gehirnveränderungen und endlich die Aetiologie des vorliegenden Falles sprechen. Bei der mikroskopischen Untersuchung der Herde erwiesen sie sich als Nester mit bindegewebigem Stroma, dessen Ausgangspunkt in den weichen Hirnhüllen und deren Gefässen zu suchen ist, weshalb sie fast alle, mit nur wenigen Ausnahmen, an der Oberfläche ihren Sitz hatten. Auffallend ist in allen diesen Herden die Gefässentwickelung; dieselben erscheinen stark geschlängelt, besitzen äusserst dicke Wände und sind stellenweise sogar obliterirt.

Fast in jedem der im Rückenmark befindlichen Herde kommen grosse Gefässe mit äusserst verdickten Wandungen, deren Stärke oft zwei- ja sogar dreimal die des Lumens übertrifft, vor (Fig. 3). Diese Veränderungen der innerhalb der Herde befindlichen Gefässe erinnerte im Allgemeinen an jene an den freiliegenden, grossen Gefässen der Gehirnbasis angetroffenen Affectionen. Auch hier hing die Verdickung der Gefässwandung hauptsächlich von der Entwicklung von Granulationselementen in der Gefässwand und von der Verdickung der äusseren in ihrem ganzen Umkreise vom straffen, fibrösen, auf ziemlich bedeutende Strecken in das umgrenzende Hirngewebe eindringenden Bindegewebe umgebenen Membran ab. Stellenweise wurden in dem Herde vollkommen obliterirte Gefässe angetroffen und in der Nähe solcher pathologisch yeränderten Gefässe concentrirte sich gewöhnlich der mit der Entwicklung von straffem, fibrösem das Mark durchsetzendem Bindegewebe einhergehende Entzündungsherd. In der Umgebung der Herde und innerhalb derselben, falls das fibröse Gewebe noch nicht seine endgiltige Entwicklung erreicht hat, finden wir eine starke Entwicklung kleiner Gefässe; dieselben erscheinen erweitert und an ihrer Peripherie sind stellenweise grosse Hengen von rothen Blutkörperchen angehäuft. Da die Herde, wie erwähnt, last überall an der Oberfläche liegen, so ist die weiche Hirnhaut in den Process selbst mit verwickelt. Mittels Bindegewebszügen verwächst sie fest mit dem darunter gelegenen Gewebe, so dass man den Eindruck erhält, als ob der Herd selbst von der weichen Hirnhaut ausgegangen wäre. Falls Nervenwurzeln in der allgemeinen Masse eines Herdes sich vorfinden, so erscheinen ihre Bündel von dickem bindegewebigem Stroma umflochten, das gleichsam eine unmittelbare Fortsetzung. der pathologisch verdickten Gefässwandungen darstellt. Die Nervenfasern unterliegen dabei in bedeutendem Masse der Degeneration mit Zerfall der Marlischeiden. Dort, wo die Gefässe weniger afficirt waren, erschien auch das angrenzende Gewebe gewöhnlich weniger sklerotisch und, im Gegentheil, in den stärker afficirten Gegenden fanden sich Gefässe, welche stark geschlängelt und fast obliterirt 
waren. Noch zu erwähnen ist, dass stellenweise in den sklerotischen Herden auch Corpora amylacea in bedeutender Menge angetroffen wurden.

Im Ganzen war der Bau der im Grosshirn befindlichen Herde derselbe (Fig. 4). Auch hier enthielt überall der Herd gewöhnlich ein oder mehrere grosse Gefässe mit äusserst verdickten Wandungen; in der Umgebung der letzteren sah man starke Entwicklung von fibrösem Bindegewebe, das nicht selten mit der mit der Gehirnsubstanz verwachsenen weichen Hirnhaut ein Ganzes bildete. Wie im Rückenmark bemerkten wir hierbei in dem angrenzenden Hirngewebe starke Gefässentwicklung und stellenweise Austritt der rothen Blutkörperchen. Die Elemente des an den Herd stossenden Nervengewebes selbst waren degenerirt. Ausserdem enthalten einige ron den Herden kleine, oft schon in der Periode der Organisation befindliche Hämorrhagien. Uebrigens besass die in der Nachbarschaft des am unteren Theile der Brücke befindlichen Herdes, wie wir gesehen, einen ziemlich bedeutenden Umfang. Die Nervengewebselemente unterliegen innerhalb der Herde der Atrophie und gehen endgültig unter. An der Herdstelle fehlen gewöhnlich die Nervenzellen ganz, da sie von bindegewebigen und Granulationselementen verdrängt werden. Dennoch erscheinea sie schon in der Nähe des Herdes als verhältnissmässig gut erhalten.

Was die Nervenfasern anbetrifft, so gehen sie den centralen Bezirken der Herde ab, in den peripheren Partien derselben sind sie aber noch erhalten, wenngleich nicht selten mit ganz offenbaren Erscheinungen der Degeneration (Fig. 3). In Bezug auf den in der Brücke vorgefundenen Herd, welcher zu einer starken Blutung in das Brückengewebe Anlass gegeben hatte, ist zu bemerken, dass auch unter dem Mikroskop sein Zusammenhang mit der pathologisch veränderten Gefässwand nachweisbar war, da das fibröse, eine beschränkte Stelle des unteren Brückentheiles einnehmende Bindegewebe hier mit der verdickten Adventitia der Basilaris ein zusammenhängendes Ganze darstellte.

Weiter wollen wir hier noch erwähnen, dass die im Rückenmark angetroffene Degeneration der Pyramidenbündel unmittelbar von dieser Hämorrhagie und den Herden in der Brücke abhängt (s. Fig. 7, 8 und 9), denn oberhalb der Brücke ist eine Degeneration der Pyramidenbündel nicht vorhanden, während die Pyramide an der Seite der Blutung aber degenerirt erscheint. Es muss ferner bemerkt werden, dass bei dor Untersuchung des Gehirns eine Affection der kleinen Gehirngefässe mit Verdickung ihrer Wandungen stellenweise sogar auch dort angetroffen wurde, wo das Firngewebe keine sichtbaren Veränderungen aufwies. Es ist klar, dass in unserem Falle die Gefässaffection eine verbreitete Erscheinung darstellte, zu pathologischen Veränderungen des Gehirngewebes aber nur dann führte, wenn sie einen mehr ausgesprochenen Grad erreicht hatte. Noch ist hier zu erwähnen, dass stellenweise in der grauen Rückenmarkssubstanz, in der Nachbarschaft des Centralkanals, freie ziemlich umfangreiche Höhlungen angetroffen werden. Da in der Nachbarschaft dieser Hohlränme ebenfalls pathologisch veränderte Gefässe mit verdickten Wandungen vorhanden waren, so wird wohl die Annahme gerechtfertigt sein, dass diese Höhlen durch die in die Rückenmarkssubstanz getretene und. wieder resorbirte Lymphe verursacht worden sind, 
In Betreff der in der Nachbarschaft des Gumma vorgefundenen Erweichung des Sehhügels und der ebenfalls in der Nachbarschaft einiger sklerotischen Herde befindlichen Erweichungsherde ist in histologischer Beziehung nichts Besonderes zu vermerken. Thre Abstammung erklärt sich zweifellos leicht durch die Ernährungsstörung der Hirnsubstanz wegen der Affection der zu den Bestandtheilen der sklerotischen Herde gehörenden Gefässe.

Schliesslich soll erwähnt werden, dass wir bei der Untersuchung im Rückenmark eine aufsteigende, secundäre Degeneration der Goll'schen Bündel im oberen Theile des Brust- und im Halsabschnitt, auch aufsteigende Degeneration des linken Kleinhirn und anterolateralen Bündels des Seitenstrangs, eine absteigende Degeneration des linken lateralen und der vorderen Pyramidenbündel in ihrer ganzen Länge (Fig. 3) und eine Degeneration des rechten lateralen Pyramidenbündels angefangen vom unteren Theile des Brustabschnittes angetroffen haben. Im verlängerten Mark und im Gehirnstamm aber wurde eine absteigende Degeneration der rechten Pyramide unterhalb des mit derBlutung in die Varolsbrücke verbundenen Herdes und ebenso eine absteigende Degeneration der linken Schleifenschicht (Fig. 5, 6, 9 und 10) eines Theiles des linken vorderen Kleinhirnschenkels und des linken centralen Haubenbündels (Fig. 8, 9 und 10) unterhalb des sklerotischen, unter dem hinteren Zweihügel befindlichen Herdes vorgefunden (Fig. 7 und 8). Ausserdem im verlängerten Marke äusserte sich die Atrophie der Hinterstrangkerne und der Formatio reticularis der rechten Seite (Fig. 11). Da wir uns mit diesen Degenerationen in der Folge specieller zu beschäftigen haben, so wollen wir nun zur Betrachtung der obenangeführten pathologisch-anatomischen Veränderungen schreiten.

Alle Untersuchungsresultate lassen uns nicht im Zweifel, dass im vorliegenden Falle es sich um eine multiple, syphilitische Erkrankung des Nervensystems handelt. Hierfür spricht nicht allein das im linken Sehhügel angetroffene Gumma und die für die Syphilis charakteristischen Affectionen der Art. basilaris, des hinteren Verbindungszweiges vom Circ. arteriosus Willisii und der Art. cerebelli ant. sup., sondern auch der histologische Bau der in so bedeutender Anzahl sowohl über das Rückenmark und die aus demselben tretenden Wurzeln, als auch über die verschiedenen Gebiete des Gehirns zerstreuten Herde. In unserem Falle nämlich hatten sich die sklerotischen Herde in der Nähe der Gefässstämme placirt, dabei stellte die Bindegewebswucherung in den Herden mit den stąrk verdickten Gefässwänden ein ununterbrochenes Ganze dar. Augenscheinlich liegt hier ein und derselbe, sowohl das Gefäss, wie das um dasselbe herumliegende Gewebe ergreifender Process vor uns. Die Frage, ob dieser Process zuerst im Gewebe sich entwickelt hatte und darauf auf die Gefässe übergegangen ist und sie mit in das Leiden hineingezogen hat, oder aber, ob derselbe von den Gefässen ausgegangen ist und sich direct auf das umgrenzende Gewebe fortgesetzt hat, muss unbedingt zu Gunsten des letzteren Entwickelungsmodus ent- 
schieden werden. Hierfür spricht erstens der Umstand, dass im gesunden Hirngewebe pathologisch veränderte Gefässe mit verdickten Wänden nicht selten angetroffen werden, und zweitens die Thatsache, dass wir hier ausserdem eine besondere, selbstständige Affection der grossen Gefässe der Gehirnbasis besitzen und drittens, dass diese Affection in einigen Fällen, wie z. B. von der Art. basilaris, sich auch auf das darunter befindliche Hirngewebe ausgebreitet hatte, da das Gefäss mit dem letzteren verlöthet war, und endlich, dass die verdickten Gefässe ausnahmslos in allen Herden vorhanden waren, wobei in einigen Fällen die stärkeren, in das Hirngewebe eindringenden Gefässe durch solche Herde hindurch traten. Es ist klar, dass bei einem anderen Verbreitungsmodus des Processes eine solche Localisation des Gefässes innerhalb des Herdes, and zwar in der Mitte desselben, ganz unverständlich bliebe. Die Veränderung selbst in den Gefässwänden der Herde kann, wie wir gesehen, ihrem Charakter nach vollkommen mit der Affection der grossen Gefässstämme an der Gehirnbasis identificirt werden. Auch hier handelte es sich um eine Verdickung der Gefässwandungen, hauptsächlich auf Kosten der Tunica adventitia, stellenweise mit Entwicklung von Granulationselementen innerhalb der Gefässwand, zwischen der Media und der Adventitia, mit Austritt von Blutkörperchen in die Umgebung der Gefässe, mit Verkleinerung der Gefässlumina und stellenweise sogar mit Obliteration derselben und mit Entwicklung von fibrösen, in das umgrenzende Gewebe eindringenden und zum allmäligen Untergange der Elemente des letzteren führenden Bindegewebe in der Umgebung des Gefässes. Besonders demonstrative Bilder lieferten die mit den sklerotischen Herden verlötheten Wurzeln. Hier war es möglich ganz deutlich zu verfolgen, wie die pathologisch veränderten Gefässe mit der Zunahme der Hyperplasie ihrer Wandungen immer mehr mit den Nervenwurzeln verwachsen, bis ein gewisser Theil der letzteren gänzlich sklerosirt war. In solchen sklerotischen Wurzeln erschienen einige von den Gefässen ganz obliterirt, an anderen waren die durch die Gefässwandung getretenen Blutkörperchen zu sehen; die Nervenfasern aber erschienen in der nächsten Nachbarschaft deutlich degenerirt und atrophisch. In Anbetracht dieser Ergebnisse der histologischen Untersuchung stellen wir in unserem Falle die eigenartige syphilitische Affection der Gefässwand in enge Beziehung zu den sklerotischen Herden, indem wir die erstere für die Ursache der Entwickelung der letzteren ansehen. Hierdurch ist die Rolle der Syphilis als des ätiologischen Moments bei der Entwicklung der sklerotischen Herde in unserem Falle deutlich nachgewiesen und wird zugleich eine positive Erklärung der anderweitig 
ganz unbegreiflichen Thatsache, dass die Krankheit im cenfralen Nervensystem inselartig verbreitet ist, gegeben.

Jedermann muss natürlich die Aehnlichkeit des von uns beschriebenen krankhaften Processes mit der gewöhnlichen disseminirten Sklerose auffallen. Die Aehnlichkeit erstreckt sich vorzüglich nur auf die äussere Seite des Processes, d. h. anf seine Verbreitung in der Form von zerstreuten Herden. Was den histologischen Bau der Herde selbst anbelangt, so unterliegt es wohl keinem Zweifel, dass in demselben ein nicht unwesentlicher Unterschied im Vergleich zu dem Bau der Herde der gewöhnlichen insulären Sklerose vorliegt. Vor Allem sind erstere ungemein derb anzufühlen, ja. erreichen sogar knorpelige Härte, was den Herden der gewöhnlichen disseminirten Sklerose nicht eigenthümlich ist. Ferner bestehen in unserem Falle die Herde aus dem um die Gefässe herum gewucherten, festen Bindegewebe, welches die Nervenelemente verdrängt und zur Degeneration und Atrophie derselben geführt hat, während bei der disseminirten Sklerose hauptsächlich Schwellung und Proliferation der Spinnen- oder Deiters'schen Zellen und Vermehrung der Neurogliazellen, nebst Wucherung des Bindegewebes vorliegt. Dabei zeigt sich eine durch Vermehrung der Kerne und Verdickung äussernde Hyperplasie der Gefässwandungen; die Nervenzellen aber unterliegen der körnigen Pigmentdegeneration, verlieren ihre Fortsätze und verwandeln sich allmälig in fortsatzlose, rundliche Körperchen, wobei einige von ihnen vacuolisirt werden. Was die Nervenfasern anbetrifft, so geht in denselben die Markscheide zweifellos verloren, während die Axencylinder meist erhalten bleiben (Charcot). Dank diesem Umstande findet man bei der gewöhnlichen disseminirten Sklerose keine secundäre Degeneration im Nervengewebe (Schulze).

In unserem Falle aber sehen wir, dass secundäre Degenerationen sowohl im Rückenmarke wie auch im Gehirne vorhanden waren. Eine von diesen Degenerationen, wie z. B. die Degeneration des rechten vorderen und linken lateralen Pyramidenbündels, muss in unserem Falle mit der Blutung in Zusammenhang gebracht werden; während die anderen Degenerationen, wie z. B. die Degeneration der Goll'schen Bündel, die Degeneration des anterolateralen Bündels und des Kleinhirnbündels und die Degeneration des Bündels im vorderen Kleinhirnschenkel, ebenso die des centralen Haubenbündels, ausschliesslich von den sklerotischen, im Verlauf dieser Bündel localisirten Herden abhängen. Ebenso wenig sind die in unserem Falle sowohl in den Herden selbst, wie in ibrer Umgebung angetroffenen Blutungen, gleichwie die Erweichungen in der Nachbarschaft einiger Herde der gewöhnlichen disseminirten Sklerose eigenthümlich; gar nicht zu reden von den für die Syphilis charak- 
teristischen Veränderungen der grossen Gefässstämme und von dem Gumma, deren Combination mit der gewöhnlichen Sklerose noch keinmal vermerkt worden ist.

Somit weist der vorliegende Fall in pathologisch-anatomischer Hinsicht so wesentliche Eigenthümlichkeiten auf, dass derselbe durchaus nicht dem Begriff rou der gewöhnlichen disseminirten Sklerose untergeordnet werden kam, vielmehr seiner Multiplicität wegen eine ganz eigenartige Affection des Nervensystems mit dem Charakter einer syphilitischen Sklerose repräsentirt.

Wir wollen nun zur Betrachtung der klinischen Seite unseres Falles übergehen.

Allgemein verlief die Remissionen und Sprünge in ihrer Entwickelung aufweisende Krankheit in unserem Falle ziemlich schnell, da bis zu ihrem Ausgang weniger als 1 Jahır und 3 Monate nöthig waren. Ihren klinischen Erscheinungen nach erinnerte sie in einiger Beziehung an die disseminirte Hirnsklerose. Bekanntlich sind in klassischen Fällen der letzteren Erkrankungsform ein charakteristisches Zittern, Nystagmus, zeitweilige Diplopie, eine besondere Erschwerung der Rede, bei welcher die Wörter silbenweise oder skandirt ausgesprochen werden, Parese der Extremitäten mit Steigerung der Sehnenreflexe und Contracturen, bei verhältnissmässig erhaltener Empfindlichkeit, welche, falls überhaupt, so doch nur zeitweilig und nicht besonders stark afficirt wird, vorhanden. Auch Blasenbeschwerden werden bekanntlich beobachtet, doch nur selten zeigt sich dieses Symptom in ausgeprägter Form. Ferner gehören zu den beständigen Symptomen: Schwindelgefühl, Intelligenzschwäche und zuweilen auch andere psychische Symptome. Zur Zahl der weniger beständigen Symptome gehören die atactischen Störungen in den Extremitäten, die Muskelatrophien und apoplectiforme Anfälle. Leider ist nicht ausser Acht zu lassen, dass die disseminirte Sklerose eine Krankheit ist, welche in gewissen Fällen wohl äusserst charakteristiche, eine genaue Diagnose erlaubende Kennzeichen vorweist, dafür aber in anderen Fällen solche Symptome nicht bietet. Ueberhaupt ist keines der angeführten Symptome absolut beständig, und es sind Fälle beschrieben, in welchen bei der Section ausgebreitete Sklerose des Gehirns und Rückenmarks vorgefunden wurde, welche aber in ihrem klinischen Verlaufe durchaus nicht typisch waren. Das sind die sogenannten Formes frustes der französischen Autoren und dieselben sollen sogar nach der Meinung einiger Forscher häufiger als die typisch verlaufenden Formen vorkommen.

Wenden wir uns nun zu unserem Falle, so sehen wir, dass hier die die typische, disseminirte Sklerose am meisten charakterisirenden Erscheinungen fehlten, so z. B. das eigenartige, pendelnde Zittern der 
Extremitäten, der Nystagmus und auch die charakteristisch articulirte oder skandirte Sprache. Doch war es aber schon bei Lebzeiten möglich auf Grund der ursprünglich aufgetretenen Schmerzen und anderen subjectiven Empfindungen in verschiedenen Körpertheilen, der Existenz einer spastischen Paraplegie ohne Erscheinungen von Anästhesie, aber mit einer Affection der Beckenorgane, der hernach sich hierzu gesellenden Diplopie, der deutlich erschwerten Aussprache von Wörtern und des Auftretens apoplectiformer Anfälle im vorliegenden Falle eine multiple Affection des Nervensystems anzunehmen. Ferner erlaubte die hernach sich auf eine zeitweilige Besserung plötzlich einstellende Paraplegie, welche mit der Zeit sich wieder verringerte, und die in der letzten Krankheitsperiode sich hinzugesellende Lähmung der rechten Hand und darauf der Kaumuskeln und die Hemianopsie gar nicht zu zweifeln, dass es sich im gegebenen Falle nicht allein um eine multiple, sondern auch um eine progressiv fortschreitende Affection des Nervensystems handelte. Unser Fall erinnert also, wenn auch nur im Grossen und Ganzen seinen klinischen Erscheinungen nach, an die atypischen Formen der disseminirten cerebrospinalen Sklerose.

Gehen wir zur Aetiologie der Erkrankung über, so können wir in unserem Falle uns nur an eine Ursache, die Syphilis, halten. Der Umstand jedoch, dass die Krankheit schon nach einem Jahre nach der Infection auftrat; muss unsere Aufmerksamkeit erregen. Diese Periode könnte zu kurz erscheinen, um in der Syphilis den Erkrankungsgrund zu sehen. Es sind aber in der Literatur Fälle von noch früherer Entwicklung der tertiären Veränderungen im centralen Nervensystem der Syphilitiker vermerkt worden. Dafür, dass wir im gegebenen Falle dennoch mit der Entwicklung von tertiären Erscheinungen der Syphilis des Nervensystems zu thun haben, spricht das im linken Sehhügel vorgefundene Gumma und die für die Syphilis charakteristischen Veränderungen der Wandungen grosser Gefässe an der Gehirmbasis. Dass selbst die Herde ihren Ursprung der Syphilis zu verdanken haben, geht nicht allein aus dem schon beschriebenen Bilde ibres Baues und ihrem Verhältniss zu den Gefässen hervor, sondern auch noch daraus, dass die antisyphilitische Behandlung zeitweilig eine unzweifelhafte und wesentliche Besserung in dem Zustande des Kranken bewirkte, obgleich sie in der Folge, bei der weiteren Entwicklung der Krankheit und Steigerung ihrer Symptome, sich als unzulänglich erwies. Somit kann es wohl keinem Zweifel unterliegen, dass die rom Patienten annähernd vor einem Jahre vor der Entstehung der gegenwärtigen Krankheit acquirirte Syphilis doch das ätiologische Moment der vorliegenden Erkrankung bildet. Das bisher Angeführte zwingt uns also zur Annahme, dass wir im 
gegebenen Falle mit einer multiplen oder disseminirten, syphilitischen Sklerose als einer besonderen Art der gewöhnlichen oder disseminirten Sklerose zu thun haben.

Die Literatur des Gegenstandes überzeugt uns vor Allem, dass die Syphilis in der Aetiologie der gewöhnlichen disseminirten Sklerose keine oder nur eine untergeordnete Rolle zu spielen scheint. Jedenfalls werdex bei der disseminirten Sklerose eine neuropathische erbliche Belastung, die Einwirkung des Kohlendunstes, das Ueberstehen verschiedener Infectionskrankheiten, der chronische Alkoholismus and andere Vergiftungen (z. B. mit Blei), Gemütbsaufregungen etc. als ätiologische Momente angeführt. Die Syphilis aber wird von einigen Autoren, wie z. B. von Gowers gar nicht unter den ätiologischen Nomenten der disseminirten Sklerose erwähnt, von anderen sogar direct aus der Aetiologie dieser Krankheit ausgestrichen. So behauptet Prof. Oppenheim, welchem wir so Vieles in der Erforschung der Syphilis des centralen Nervensystems verdanken, direct, dass „die multiple Sklerose mit der Syphilis nichts gemein hat ${ }^{l *}$ ). Andere Autoren weisen übrigens dje Syphilis nicht so categorisch aus der Aetiologie der disseminirten Sklerose zurück. Strümpell**) z. B, zählt die Frage, ob Syphilis als Ursache der multiplen Sklerose anzusehen sei, zu den streitigen. Schuster hat fermer einen Fall von disseminirter Sklerose beschrieben, welche mit der Syphilis zusammenhing und durch antiluetische Behandlung geheilt wurde. Endlich ist Moncorvo**) in letzterer Zeit auf Grund von vier eigenen Beobachtungen mit Entschiedenheit für die Möglichkeit einer Entwicklung der disseminirten Sklerose bei Kindern unter dem Einfluss hereditärer Syphilis eingetreten. $Z u$ derselben Auffassung neigt sich Prof. Philatow + ) bei der Analyse einer Beobachtung an einem Kinde mit ausgesprochenem bulbären Symptomencomplex und spastischem Zustand der unteren Extremitäten. Andererseits finden wir in der Literatur disseminirte sklerotische Affection des Nervensystems syphilitischen Ursprungs, welche mit der disseminirten Syphilis grosse Uebereinstimmung bietet, beschrieben. Vor Allem wollen wir eine obgleich nur pathologisch-anatomisch interessante Beobachtung von Charcot und Gombault (Archiv. de phys. 1873, Vol. II, pag. 173) anführen. Darüber lesen wir bei Gowers $\dagger$ ): „Eine sehr seltene Modification von

*) Gowers, Handb. der Nervenkrankh. Bd. II. 1892. p. 539-540.

*) Strümp ell, Krankheiten des Nervensystems. Leipzig, 1889.

***) Moncorvo, Revue mens. des maladies de l'enfance. 1887. t. V.

†) Prof. Philatow, Medicin. Vebersicht No. 1, 1894. p. 32 u. s. w.

+f) Gowers, Handb. der Nervenkrankh. Bd. II. p. 465. 
Cerebritis repräsentirt die disseminirte Form der chronischen sklerotischen Entzündung, welche augenscheinlich als eine Folge der Syphilis erscheint. In dem von Charcot und Gombault beschriebenen Falle ... existirten ähnliche Herde in der Brücke, in den Hirnschenkeln und in den Sehnerven... die Herde erschienen grau mit gelblichen Centren in Folge der Verkäsung. Beide Abducenten und der rechte Oculomotorius erschienen theilweise degenerirt. Durch seine Neigung zur Verkäsung charakterisirte sich der Process als syphilitische Neubildung, während die histologischen. Eigenthümlichkeiten mehr der skerotischen Entzündung als der Neubildung eigen waren. In diesen Punkten bot der Process grosse Uebereinstimmung mit den Herden bei der chronischen syphilitischen Meningitis dar. Vor der Erkrankung an Syphilis war der Kranke ungefähr 20 Jahre alt gewesen. Die bei ihm vorhandene starke Intelligenzschwäche verdunkelte einen grossen Theil der Symptome der intracraniellen Affection. Eine solche zerstreute Entzündung ist andererseits mit der disseminirten Sklerose verwandt". Derselbe Autor spricht ferner auf der 548. Seite: „Eine andere mit der multiplen Sklerose grosse Uebereinstimmung aufweisende Erkrankung ist die disseminirte, sklerotische Entzündung syphilitischen Ursprungs im Gehirn und Rückenmark. Beide Processe verlaufen gleich chronisch und ihre Vertheilung ist eine annähernd gleiche. Es ist zweifelhatft, ob ihr histologischer Charakter Anhaltspunkte zu ihrer Unterscheidung liefern wird. Ein wichtiger Unterschied ist durch den Fund von käsigen Knoten bei der syphilitischen Erkrankung gegeben, da solche bei der disseminirten Sklerose fehlen".

Mit Ausnahme des in klinischer Hinsicht leider ungenügend verfolgten Falles von Charcot und Gombault sind diese Angaben übrigens so allgemein, dass eine Ausscheidung der disseminirten, syphilitischen Sklerose als eine besondere Form der Erkrankung bisher wegen Mangel an entsprechenden Beobachtungen nicht zu begründen war. Es muss hier jedoch erwähnt werden, dass Kahler*) geneigt ist, in der multiplen, syphilitischen Wurzelneuritis eine selbstständige Erkrankungsform zu sehen, wobei er sich auf eine Beobachtung von Buttersack ${ }^{*}$ ) und einen eigenen Fall, in welchem hauptsächlich cerebrale Nerven und spinale Wurzeln ergriffen waren und die weichen Hirnhüllen eine geringe Betheiligung aufwiesen, stützt. Da in unserem Falle zugleich mit der

*) Kahler, Vierteljahrsschrift Bd. VIII. 1887. Vergl. Oppenheim, Zur Kenntniss der syphilitischen Erkrankungen des centralen Nervensystems. Berlin 1890.

**) Dieses Archiv Bd. XVII, 1886. 
syphilitischen Affection der Nervenwurzeln eine grosse Anzahl disseminirter Herde im Hirngewebe sellsst vorhanden waren, so muss dieser Erkrankungsfall als syphilitische, disseminirte, cerebrospinale Sklerose angesehen werden und die multiple, syphilitische Wurzelneuritis von Kahler repräsentirt nur eine Abart, oder richtiger gesagt, einen einzelnen, speciellen Fall derselben. Wenigstens sehe ich keine triftigen Gründe zur Erhebung der von Buttersack und Kahler beschriebenen Fälle zu einer besonderen Erkrankungsform. Sowohl in denselben wie in unserem und ebenso in dem Falle von Charcot und Gombault handelt es sich nämlich um einen multiplen, syphilitischen, neoplastischen, die verschiedensten Abschnitte des centralen Nervensystems, mit Einschluss der aus demselben hervortretenden Wurzeln befallenden Process. Die unter der Bezeichnung einer syphilitischen, gummösen Neuritis beabsichtigte Ausscheidung der Fälle von Buttersack und Kahler in eine besondere Categorie kann schon deshalb nicht gestattet werden, weil es sich in diesen Fällen nur um eine Wurzelneuritis, folglich um eine Affection der Nervenstämme noch innerhalb der cerebrospinalen Höhle und nicht ausserhalb derselben handelt, und dabei auch die Hüllen, wemn auch im geringen Grade, vom Process mitergriffen waren. Andererseits war sowohl in dem Fall von Charcot und Gombault, wie auch in dem unserigen, zugleich mit den disseminirten Herden im Hirngewebe und unter einiger Betheiligung der Hüllen auch eine gummöse Affection der Wurzeln von ganz demselben Charakter, wie in den Fällen von Buttersack und Kahler, vorhanden. Hieraus geht doch klar hervor, dass in den letzteren Fällen der Process entweder zufälligeryeise hauptsächlich die Wurzeln befallen hatte, oder aber noch nicht den in unserem Falle vorhandenen Enwickelungsgrad errreicht hatte, wo ausser den Wurzeln auch das Hirngewebe und in geringem Grade auch die Hüllen schon afficirt waren. Es erscheint mir ferner, dass man sich auch durchaus nicht mit der Meinung von 0 ppenheim, dass es sich in diesen Fällen um eine primäre Affection der weichen Hirnhäute handele, von wo aus der Process auf die Wurzeln übergegriffen habe, für einverstanden erklären kann. Um den Standpunkt Oppenheim's zu verstehen, müssen wir zuerst näher auf die Frage über die besondere, von diesem Autor unter der Bezeichnung Meningitis syphilitica cerebrospinalis ${ }^{*}$ ) beschriebenen Form einer syphilitischen Affection des Nervensystems eingehen.

*) Oppenbeim, Zur Kenntniss der syphilitischen Erkrankungen des centralen Nervensystems. Berlin 1890. 
Vor Allem wollen wir hier den von Oppenheim als Beispiel dieser Erkrankungsform beschriebenen Fall kurz angeben:

Die 24jährige, sieben Jahre vor der gegenwärtigen Erkrankung von Syphilis inficirte Kranke leidet drei Jahre schon an einer Dickdarmverengerung specifischen Ursprungs. Sie giebt an, vor drei Monaten Schwäche in den Füssen gefühlt zu haben, welche schnell zugenommen und den Grad einer Jähmung erreicht habe; hierzu wären in den letzten Tagen Herabsetzung der Sensibilität, Gürtelgefühl und Erschwerung der Harnentleerung hinzugekommen. Ueber cephalische Beschwerden klagte die Patientin nicht, aber Andere behaupteten, dass sie sich schon seit fünf Jahren in psychischer Beziehung verändert habe und theilnahmslos und apathisch geworden sei. Weiter stellte es sich heraus, dass einige Wochen vor der Aufnahme, nämlich im Juni desselben Jahres, die Kranke an Diplopie und, wie es scheint, auch an Accommodationsparese gelitten hatte. Bei der Untersuchung wurde bedeutende Schwäche der tunteren Extremitäten, hauptsächlich der linken, mit krampfhaften Erscheinungen vorgefunden; die Sensibilität ersehien nur an der rechten Tibia und am rechten Fussgelenk gestört, wo Kälte als Wärme empfunden wurde. Die Kranke hatte häufiges Bedürfniss zu uriniren und musste bei der Harnentleerung drängen; zu gehen war sie nicht im Stande. In den oberen Extremitäten existirte nur Schwäche und geringes Zittern bei den Bewegungen. Zugleich bestand eine geringe, vomAutor besonders vermerkte Differenz in der Pupillenweite, eine geringe und in ihrer Intensität schwankende, linksseitige Ptosis und schlaffer Lichtreflex der linken Pupille. Anfangs erhielt die Kranke Jodkalium, dann aber, als die Existenz einer stattgefundenen, syphilitischen Infection festgestellt war, wurde eine energische Behandlung mit Quecksilberinjectionen eingeleitet und zwar mit auffallendem Erfolg: nach Verbrauch von $50 \mathrm{Grm}$. konnte die Kranke wieder stehen und gehen; die Behinderung der Harnentleerung war ebenfalls verschwunden, gleichwie das Gürtelgefühl und die Sensibilitätsstörungen. Doch hatte man es nur mit einer Remission der Krankheit zu thun, denn trotz der Fortsetzung der Behandlung wurden die Lähmungserscheinungen mit der Zeit stärker, wozu sich hernach noch eine in ihrer Ausbildung schwankende, bald am rechten Fuss, bald wieder am linken, stärker ausgesprochene Ataxie und starke Behinderung der Harnentleerung, hinzugesellte. Ausserdem stellten sich temporär allgemeine cerebrale Symptome: Kopfschmerz, Schwindelgefïhl und Erbrechen ein. Anfang Januar wies der Augenspiegel eine geringe Veränderung des Augenhintergrundes und deutlichere Lähmungserscheinungen am linken Auge nach. Den 22. Januar verlor die Kranke plötzlich die Besinnung, war beinahe pulslos, cyanotisch und zeigte das Cheyne-Stokes'sche Athmungsphänomen, welcher Zustand einige Stunden anhielt. Von dieser Zeit ab fing die Lähmung der Füsse an zuzunehmen, das Gürtelgefühl wurde wieder stärker, die Störung der Empfindlichkeit überhaupt und die des Temperatursinnes erstreckte sich auf beide Unterextremitäten, war dabei aber rechts stärker ausgesprochen als links; zu gleicher Zeit wurde auch die Schwäche der oberen Extremitäten deutlicher, es trat 
Decubitus, Pulsbeschleunigung und kurze Zeit darauf Parästhesie nnd Empfindlichkeitsabnahme in der Trigeminusgegend und vollständige Lähmung des rechten Facialis mit Betheiligung seines oberen Zweiges auf. Drei Tage nach der Ausbildung dieser Lähmung wurde sie wieder comatös; es trat Lungenentzündung hinzu und die Kranke verschied den 3. Februar 1889. Während der Krankheit war Fieber nicht zugegen gewesen. Vom Autor war die Diagnose auf Meningitis gummosa cerebrospinalis gestellt worden. Die Obduction ergab Folgendes: An der Gehirnbasis Verdickung und Trübung der weichen Hirnhüllen, besonders in der Umgebung des Chiasma; in der Brückengegend and seitlich von ihr eine eigenartige, sulzige, bis zum verlängerten Mark hinziehende Infiltration der weichen Hirnbaut. Dieses neoplastische, gefässreiche Gewebe bedeckt auch theilweise die Anfangstheile der Nerven. Bei einer genaueren Untersuchung erwies es sich, dass ein Theil der letzteren, besonders die Sehnerven, der linke Oculomotorius, der rechte Facialis, Acusticus und die Vagi verdickt und mit der Neubildung verwachsen sind, und auf der Durchschnittsfläche grünlich gefärbt erscheinen. Die Wandungen der grossen, an der Gehirnbasis befindlichen Gefässe, nämlich der Basilar- und Vertebralarterien sind verdickt, und ihre äussere Membran lässt sich kaum von der Neubildung trennen; die Basilaris erscheint wegen einer starken Intimaverdickung bedeutend verengt und ihre Lichtung ist mit Gerinnseln angefüllt. Als sehr stark afficirt erwies sich auch die Art. corp. callosi. In den Gehirnventrikeln mässiger Hydrops. Keine Herdprocesse. Derselbe Process in sehr starler Entwickelung war auch am Rückenmark vorhanden. Dasselbe war gigantisch vergrössert und verdickt und bei dem Versuch, die harte Hirnhaut zu entfernen, wurde bemerkt, dass dieselbe in den oberen zwei Dritteln mit der Arachnoidea gänzlich verwachsen war. Die Hüllen bedentend verdickt und durchdrungen theils von einer weichen gallertigen, an anderen Stellen aber von einer sehr derben Gewebsmasse. Das Rückenmark selbst ist seiner ganzen Länge nach verändert; Seine Substanz besitzt an vielen Stellen eine gelbliche Farbe; ihre Contouren sind verwaschen. Das erweichte Gewebe zeigt unter dem Mikroskop zahlreiche runde und körnige Zellen. Eine mehr ausgesprochene Erweichung wird am unteren Hals- und oberen Brustabschnitt bemerkt. Die spinalen Wurzeln sind von der Neubildung eingeschlossen und erscheinen theils von glasartig grauer Farbe. Nach unten und hinten verringert sich die Affection allmälig; im Lendentheil aber sind die Hüllen stark injicirt. An anderen Organen auffallend geringe Veränderungen. Die pathologisch-anatomische Diagnose lautete: Syphilis constitutionalis, Arachnitis cerebrospinalis syphilitica, Myelomalacia flava punctata, Atrophia granularis renum, Strictura recti, Hypertrophia et Dilatatio coli partialis.

Von den Resultaten der mikroskopischen Untersuchung verdient nach dem Autor der Umstand Beachtung, dass das Chiasma im vorderen Winkel Veränderungen, wenn auch geringfügige, aufwies, trotzdem bei Lebzeiten Sehstörungen gefehlt hatten. Ferner wurde eine typische, gummöse Neuritis der Nn. oculomotorii, besonders des linken und ebenso 
des Facialis, Abducens und des Vagus vorgefunden. Ausserdem fand sich eine Atrophie der rechten aufsteigenden Trigeminuswurzel.

Der Autor hält seinen Fall für eine typische, syphilitische, cerebrospinale Meningitis. Bei der Beurtheilung derselben findet er, dass ein in einiger Hinsicht gleicher Fall von Buttersack*) mitgetheilt worden sei, in welchem Falle aber die cerebralen Symptome mehr in den Vordergrund getreten seien, während die Affection des Rückenmarks, von welcher vorzüglich die Wurzeln ergriffen gewesen seien, sich hauptsächlich durch Reizerscheinungen geäussert habe. Der Autor zählt hierher auch die ihres acuten Verlaufes wegen interessante Beobachtung von $\left.K n a p p^{* * * x}\right)$. Seiner Meinung nach stellt auch die Beobachtung von Ziemssen ${ }^{* *}$ ), jedoch nur in klinischer Hinsicht einen typischen Fall von cerebrospinaler Meningitis dar. Endlich führt der Autor bei der Beurtheilung seines Falles auch den von Kahler unter der Bezeichnung einer syphilitischen Wurzelneuritis beschriebenen Fall an.

Als Argument gegen die Aufstellung derartiger Fälle als besondere Erkrankungsformen wird von ihm der Umstand in's Feld geführt, dass es unmöglich wäre, den Einwand zu beseitigen, dass auch in diesem Falle die weichen Hirnhüllen den primären Affectionsort abgegeben hätten, und der meningitische Process sich möglicherweise nur in Folge der Behandlung zurückentwickelt habe, während die stärker afficirten cerebralen und spinalen Wurzeln unverändert geblieben seien. Die Untersuchung unseres Falles lässt aber keinen Zweifel darüber zu, dass der Process sich hauptsächlich in den Gefässen entwickelt hat und nicht von den Hüllen ausgegangen ist, da hierfür nicht allein die rerhältnissmässig schwache Betheiligung der Hirnhüllen an dem Processe selbst, sondern auch das ganze Resultat der mikroskopischen Untersuchungen spricht. Das letztere demonstrirte ad oculos, dass überall, die Wurzeln nicht ausgenommen, der Process in der Umgebung ler ausgesprochene Bilder einer Affection aufweisenden Gefässe sich entwickelt hatte. In gleicher Weise dient der Umstand, dass in unserem Falle die Herde nicht allein an der Oberfläche, sondern auch in der Tiefe der Gewebe (z. B. unter dem Vierhügel) sich befanden, als Beweis, dass der Hauptausgangspunkt des Processes selbst die Gefässe und nicht die Hirnhüllen selbst gewesen sind. Mit gutem Grunde kann angenommen werden, dass die Betheiligung der letzteren an der Affection in hohem Grade

*) Buttersack, Dieses Archiv Bd. XVII. 1886.

**) Knapp, Neurol. Centralbl. No. 4. No. 4.

***) r. Ziemssen's, Klin. Vorträge. Nervensystem No. 3. Die Syphilis des Nervensystems. Leipzig 1888. 
von ihrem Reichthum an Gefässen abhängt und dass letztere wenigstens dort, wo es sich um Herdprocesse handelt, primär afficirt werden. Ebenso ist in unserem Falle die auffallend starke Betheiligung der Wurzeln an der Affection angenscheinlich von ihrem ziemlich bedeutenden Gehalt an arteriellen Gefässen abhängig. Für diese Meinung sprechen, wenigstens in unserem Falle, mit Entschiedenheit die Resultate der mikroskopischen Untersuchungen, weil überall, wo die Wurzeln sich als sklerosirt erweisen, in den sklerotischen Herden ein grösseres oder kleineres, den Ausgangspunkt der Affection bildendes Gefäss nachweisbar war. Natürlich wollen wir damit nicht behaupten, dass in unserem Falle die diffuse Affection der weichen Hirnhüllen an der Gehirnbasis sich nicht primär entwickelt hat; diese gleichzeitig vorhandene, diffuse Affection der weichen Hüllen erschien jedoch verhältnissmässig gering und konnte keinesfalls den Grundton der Erkrankung verdecken. Noch mehr: Würden wir bei unserem Falle an dem Standpunkte Oppenheim's festhalten, so könnten wir uns gar nicht den Umstand erklären, warum der Process gerade im gegebenen Falle nicht einen diffusen Charakter besitzt, sondern als eine disseminirte Herdaffection, welche die Hirnhüllen, die Wurzeln und das Hirngewebe selbst ergreift, erscheint. Sehen wir dagegen die Hirngefässe als den Ausgangspunkt des krankhaften Processes an, so erhalten wir eine vollkommen genügende Erklärung für eine solche Verbreitung des Processes und können in dieser Hinsicht denselben den diffusen, syphilitisehen Affectionen der weichen Hirnhüllen gegenüberstellen, gleichgiltig, ob eine solche Affection an der Gehirnbasis oder an seiner convexen Oberfläche, oder endlich im Rückenmarke localisirt ist.

Somit zeigt also die Gegenüberstellung der Fälle von Charcot und Gombault, Kahler und von mir in pathologisch-anatomischer Beziehung, dass es sich in denselben um disseminirte Herde mit Neubildung von Bindegewebe handelt, welche an den Wurzeln, Hüllen und im Hirngewebe selbst, oder vorwiegend nur an irgend einem dieser Theile localisirt sein können. Folglich besitzen wir hier das Bild einer disseminirten, syphilitischen Sklerose, deren Ausgangspunkt, wie es der von uns untersuchte Fall und die nähere Analyse der von anderen Autoren beschriebenen beweist, die von der Affection sogar dort, wo sie innerhalb der Schädelhöhle vollkommen frei, $d$. h. ausserhalb der Hirnsubstanz liegen, nicht verschonten Gefässe bilden. Kurzum, auf Grundlage der oben angeführten Fälle kann uns das Recht in pathologisch-anatomischer Hinsicht eine syphilitische disseminirte, cerebrospinale Sklerose (Sclerosis disseminata cerebrospinals syphilitica), folglich eine nach ihrer Localisation der gewöhnlichen disseminirten, cerebrospinalen Sklerose 
sehr gleichenden, von derselben aber durch den Charakter des krankhaf ten Processes selbst sich unterscheidende Erkrankungsform aufzustellen, nicht abgesprochen worden. Existirt also in pathologiseh-anatomischer Hinsicht eine besondere Form des krankhaften Processes, welche wir als syphilitische disseminirte Sklerose bezeichnen, so entsteht die Frage, ob sie in ihrem Krankheitsbilde solche klinische Kennzeichen bietet, welche sie schon am Krankenbette za erkennen und von der gewöhnlichen disseminirten Sklerose zu unterscheiden erlaubt. Zweifellos ist diese Frage von eminent practischer Bedeutung, aber ebensowenig unterliegt es einem Zweifel, dass zu ihrer Entscheidung noch weitere Beobachtungen nothwendig sind. Trotzdem halten wir es jedoch nicht für überflüssig, hier einige Besonderheiten der in Rede stehenden Krankheitsform zu vermerken, welche sie auch klinisch erkennbar machen und von der gewöhnlichen disseminirten Sklerose zu unterseheiden erlauben.

Diese Eigenheiten treten schon bei der Analyse unseres Falles klar hervor. Schon oben haben wir erwähnt, dass unser Fall nach den kli nischen Anzeichen ziemlich stark an die atypischen Fälle der disseminirten Sklerose erinnert; trotzdem aber sind an demselben einige klinische Eigenheiten kaum zu verkennen, welche, falls sie uiberhaupt bei der gewöhnlichen, wenn auch atypischen Form der Sklerose zur Beobachtung gelangen, so jedenfalls keine beständige Erscheinung derselben bilden. Von den spinalen Erscheinungen verdiente vor Allem Beachtung der bei dem Patienten schon während der ersten Entwickelung der Krankheit vorhandene paretische Zustand der unteren Extremitäten, welcher ziemlich schnell in eine wirkliche Paraplegie überging. Hierbei zeigte auch der Gang des Patienten vou Anfang an, zugleich mit den spastischen Erscheinungen, einen deutlichen paretischen Charakter und kömnte als paretisch-spastisch bezeichnet werden.

Bei der disseminirten Sklerose aber treten, besonders zu Anfang der Erkrankung, weniger die Erscheimungen der Parese, als eine durch das Zittern bewirkte Bewegungsstörung in den Vordergrund. Hierzu kommt, dass die Lähmungserscheinungen fast niemals den Grad einer ausgesprochenen Paraplegie erreichen, sondern sich nur als Parese der Extremitäten äussern.

Wir möchten noch auf die frühe, durch Störungen der Blaseufunction sich äussernde Affection der Beckenorgane in unserem Falle aufmerksam machen. Wohl wird auch bei der wirklichen Sklerose eine Affection der Beckenorgane beobachtet, jedoch am häufigsten in viel späteren Krankheitsperioden, während das frühe Auftreten dieses Symptoms bei der wirklichen disseminirten Sklerose zu den grössten Seltenheiten zu gehören scheint. Es verdienen ferner Beachtung die bei un- 
serem Patienten schon ganz zu Anfang der Krankheit auftretenden Parästhesien, welche ebenfalls bei der wirklichen Sklerose selten beobachtet werden. Eine weitere, in unserem Falle zur Beobachtung gelangte Eigenheit, welche unserer Meinung nach ebenfalls einige Beachtung verdient, besteht in der localen Empfindlichkeit der Wirbelsäule gegen Percussion. Dieselbe bildet nach meinen Beobachtungen ein gewöhnliches Symptom der syphilitischen Rückenmarksaffection, besonders der von Erb unter der Bezeichnung einer syphilitischen Spinalparalyse*) aufgestellten Form, während es bei der wirklichen Sklerose in der Literatur noch nicht vermerkt worden ist. Das wären die spinalen Erscheinungen, welche unserer Ansicht nach unseren Fall von den Fällen einer wirklichen disseminirten Sklerose unterscheiden.

Wünscht man einen Vergleich, so verhält sich unser Fall der syphilitischen disseminirten Sklerose, bezüglich der spinalen Symptome, zu der wirklichen disseminirten Sklerose annähernd ebenso, wie die syphilitische Spinalparalyse zur Lateralsklerose.

Wir haben ferner schon oben erwähnt, dass in unserem Falle das für die wirkliche Sklerose so charakteristische, eigenthümliche Zittern der Extremitäten und des Kopfes, die skandirende Sprache und der Nystagmus micht vorhanden waren. Wir wissen aber, dass diese, sonst so charakteristischen Erscheinungen auch bei der wirklichen disseminirten Sklerose nichts weniger als beständig sind. Trotzdem glauben wir, dass es sehr wünschenswerth ist, diesen Symptomen bei den weiteren Beobachtungen von syphilitischer disseminirter Sklerose besondere Aufmerksamkeit zu schenken. Es könnte sich herausstellen, dass sie in diesem Falle überhaupt nicht zur Beobachtung gelangen, oder aber eine seltene Ausnahme bilden. Wenigstens habe ich dieselben bei meinen klinischen Beobachtungen von disseminirter Sklerose, bei welcher die Syphilis eine hervorragende Rolle in der Aetiologie spielte, bisher nie angetrofien. Bisweilen war wohl auch in solchen Fällen Zittern der Extremitäten und Erschwerung der Aussprache von Wörtem, gleichwie in dem hier beschriebenen Fall zugegen, jedoch ohne jene, die wirkliche disseminirte Sklerose auszeichnenden Eigenthümlichkeiten. Das hier etwa vorkommende Zittern ist kurz und tritt meist in den Fingern bei ihrer Ausstreckung, d. h. wie jenes bei der Anspannung der Muskeln, auf. Ebenso erscheint die Sprachstörung in der Form einer eimfachen, dysarthrischen

*) Leider hat dieses Symptom bisher noch wenig Berücksichtigung gefunden, ist aber unter Anderem auch in der Hinsicht von Bedeutung, dass es annähernd genau das Niveau der Rückenmarksaffection zu diagnosticiren erlaubt. (Vergl. meinen Artikel.) 
Störung, welche sich durch erschwerte und nicht ganz deutliche Aussprache der Wörter kundgiebt und nicht jene gleichmässige, durch eine eigenartige Articulation der Silben zu Stande kommende, für die wirkliche disseminirte Sklerose so charakteristische Verlangsamung der Rede aufweist. Was die Augensymptome anbetrifft, so bestanden dieselben in unserem Falle in zeitweiligem Schielen und in der Diplopie, welche sich schon ganz zu Anfang der Erkrankung einstellte, was meiner Meinung nach für die Diagnose ebenfalls nicht ganz gleichgültig ist. Kurz vor dem Tode endlich zeigte sich auch eine Hemianopsie.

Soweit mir die Beobachtungen anderer Fälle von syphilitischer disseminirter Sklerose zu urtheilen gestatten, gelangen die Augensymptome oft und dabei in frühen Perioden der Krankheit zur Beobachtung und bestehen meist in Parese der Augenmuskeln. Keinmal habe ich jedoch in der oben angeführten Beobachtung ähnlichen Fällen den atactischen, sich durch Augapfelschwankungen äussemden, für die wirkliche disseminirte Sklerose so charakteristischen Nystagmus zu vermerken gebabt.

In Bezug auf den Krankheitsverlauf muss in unserem Falle die schroffe, schubweise Entwickelung des krankhaften Processes mit Schwankungen nach beiden Seiten hin die Aufmerksamkeit auf sich ziehen. Wohl entwickelt sich auch bei der wirklichen disseminirten Sklerose die Krankheit schubweise, es macht sich aber dabei bei jedem Schub der Krankheit ein constanter, den krankhaften Process fast unaufhaltsam weiter rückender Fortschritt bemerkbar, und wenn Remissionen auch bei dieser Krankheit möglich sind, so erscheinen dieselben augenscheinlich im Allgemeinen nicht erheblich. Bei der von uns beobachteten form aber sehen wir schroffe, schubweise Schwankungen des krankhaften Processes: bald tritt eine plötzliche Recrudescenz, dann wieder eine Remission und abermals wieder eine Verschlimmerung ein. Mit der Zeit progressirt aber natürlich auch diese Erkrankungsform.

Nicht geringere Beachtung verdient in unserem Falle anch der auffallend acute Verlauf der Krankheit. Im Allgemeinen setzte die Krankheit in den ersten Tagen des August 1888 ein und endete mit dem Tode am 20. October 1889, folglich erforderte sie bis zu ihrem Ausgange nicht über $1 \mathrm{Jahr}$ und 3 Monate. Nun wissen wir aber, dass die wirkliche disseminirte Sklerose im höchsten Grade chronisch verläuft und sich viele Jahre hindurch hinzieht. Es ist vorauszusetzen, dass unser Fall in dieser Hinsicht gewissermassen eine Ausnahme bildet, obgleich derselbe auch nicht ganz isolirt in der Literatur dasteht. Wir sahen, dass der Fall Knapp sich ebenfalls durch einen auffallend acuten Verlauf auszeichnete. Wenn die syphilitische disseminirte Sklerose in anderen Fällen auch nicht so acut verläuft, wie in unserem, so lässt sie doch 
hinsichtlich der Schnelligkeit, mit welcher ihre Symptome sich entwickeln, die Fälle von wirklicher disseminirter Sklerose weit hinter sich zurück.

Beachtenswerth ist endlich, dass die antiluetische Behandlung die syphilitische disseminirte Sklerose einigermassen günstig beeinflusst. $\mathrm{Zu}$ Anfang der Krankheit wurden in unserem Falle nach einer energischen, antisyphilitischen Behandlung die Krankheitssymptome bedeutend schwächer. Obwohl sie sich mit der Zeit wieder einstellten und hierauf beständig progressirten, so dürfte davon bis zu einem gewissen Grade doch anch die Nachlässigkeit des Patienten in Bezug auf seine Behandlung die Schuld tragen; denn nach der erhaltenen Erleichterung stellte er dieselbe ein, trotzdem die Fortsetzung der Behandlung klar angezeigt und ihm dringend angerathen wurde. Uebrigens ist nicht ausser Acht zu lassen, dass die Fälle von syphilitischer disseminirter Sklerose überhaupt eine bei Weitem nicht günstige Voraussage zuzulassen scheinen, und wenn es auch gelingen mag, sie zu heilen, wie der Fall von Schuster beweist, so geschieht solches nicht oft, wenigstens in der Periode der vollen Entwickelung der Krankheit.

Es gehören somit zu den Besonderheiten unseres Falles und unterscheiden denselben von der gewöhnlichen disseminirten Sklerose: 1. die Abwesenheit solcher Hauptsymptome der disseminirten Sklerose wie der Nystagmus und das schwankende Zittern der Glieder; 2. die acute Entwickelung der Paraparese und Paraplegie in der Anfangsperiode der Krankheit; 3. das frühe Auftreten der Functionsstörungen der Beckenorgane, welche sich durch ausgesprochene Behinderung der Harnentleerung äussert; 4. das frühe Auftreten der verschiedenartigen Parästhesien und der neuralgischen Gefühle in den Extremitäten und am Rumpfe; 5. die locale Empfindlichkeit der Wirbelsäule gegen Percussion; 6. das frühe Auftreten paretischer Erscheinungen an den Augen. 7. der schubweise Verlauf der Krankheit mit schroffen Remissionen und Verschlimmerungen der Symptome; 8. die deutliche Besserung der Krankheit in Folge einer antisyphilitischen Behandlung; 9. die Schnelligkeit der Entwickelung der Symptome und der acute Verlauf der Krankheit und 10. die Syphilis in der Anamnese, unter den wichtigsten ätiologischen Momenten.

Der Vergleich unseres Falles mit anderen in der Literatur beschriebenen Fällen ähnlicher Art ergiebt, dass den aufgezählten Unterscheidungsmerkmalen eine mehr allgemein gültige Bedeutung zukommt, und dass dieselben nicht nur als Besonderheiten eines speciellen Falles angesehen werden können. $\mathrm{Zu}$ hoffen ist, dass mit der Zeit, wenn eine grössere Zahl von Beobachtungen der Fälle syphilitischer, disseminirter, cerebrospinaler SkIerose veröffentlicht worden ist, noch andere differen- 
tialdiagnostisch verwerthbare Symptome angegeben werden. Nichtsdetoweniger glaube ich auf Grumd unseres Falles annehmen zu können, dass an der Hand der angegebenen Kennzeichen wir schon jetzt in den Kliniken im Stande sind, diese Erkrankungsform von der gewöhnlichen disseminirten, cerebrospinalen Sklerose zu unterscheiden.

Es entsteht jedoch die Frage, ob es möglich ist, nach den klinischen Symptomen die syphilitische, disseminirte, cerebrospinale Sklerose von der syphilitischen cerebrospinalen Meningitis Oppenheim's zu unterscheiden. Zweifellos sind beide Erkrankungsformen noch nicht dermassen studirt worden, dass es schon jetzt möglich wäre, sich mit voller Gewissheit über die Unterscheidungsmerkmale beidex Krankheiten zu äussern. Dennoch aber kann ich, wenn ich mich daran halte, was wir in Bezug auf die Erscheinungen und den Verlauf der syphilitischen, cerebrospinalen Meningitis Oppenheim's wissen, darauf hinweisen, dass bei derselben gewöhnlich, in mehr oder weniger hohem Grade, allgemeine cerebrale Symptome, als starke Kopfschmerzen, Schwindelgefühl und Erbrechen, und ebenso auch psychische Störungen, als Intelligenzschwäche, Apathie und vorübergehende Besinnungsverluste auftreten. Aller Wahrscheinlichkeit nach ist auch das frühe Auftreten der Symptome seitens der cerebralen Nerven bei der syphilitischen cerebrospinalen Meningitis nicht ohne Bedeutung, besonder's wenn dabei einige cerebrale Nerven afficirt sind. Die gekreuzte Hemiplegie, als ein ziemlich häufiges Symptom der syphilitischen Basilarmeningitis, kann, wie es scheint, ebenfalls eine differentialdiagnostische Rolle spielen und für eine syphilitische cerebrospinale Meningitis sprechen. Von den spinalen Symptomen können zur Unterscheidung der syphilitischen cerebrospinalen Meningitis von der syphilitischen cerebrospinalen Sklerose besonders folgende, bei der ersteren zur Beobachtung gelangenden Symptome dienen: deutliches Gürtelgefühl, ausgesprochene seuralgische Empfindungen und ebenso mehr ausgebildete Veränderungen der Sensibilität, sowohl der allgemeinen wie des Temperatursinnes, und die der Brown-Séquard'schen Parese oder Paralyse entsprechenden Erscheinungen Der Verlauf der Krankheit ist in beiden Fällen ein schubweiser, mit starken Remissionen und Exacerbationen, weshalb derselbe nicht bei der Differentialdiagnose verwerthet werden kann.

Bevor wir die oben beschriebene Beobachtung schliessen, müssen noch einige Worte über die durch die sklerotischen Herde verursachten secundären Degenerationen der Hirusubstanz verlautet werden.

Von diesen secundären Degenerationen müssen vermerkt werden: die ascendirende Degeneration der Goll'schen Bündel an dem Brustund Halsabschnitt des Rückenmarks, die descendirende Degeneration der 
linken Pyramide und der rechten lateralen und beiden vorderen Pyramidenbündel des Rückenmarkes. Ferner zeigt sich in unserem Falle im unteren Theil des Brustabschnittes vom Rückenmarke eine descendirende Degeneration des Pyramidenbündels nicht allein im linken, sondern auch im rechten Seitenstrange; augenscheinlich war diese Degeneration durch den sklerotischen, am Brustabschnitt des Rückenmarks sitzenden Herd verursacht worden. Endlich fanden wir in unserem Falle in der Gegend des Hirnstammes eine secundäre Degeneration folgender Bündel vor: 1. eine absteigende Degeneration eines kleinen Bündels des oberen oder vorderen Kleinhirnschenkels, welche sich von dem beschriebenen, in der Haube, unter dem hinteren Zweihügel befindlichen Herd in der Richtung des Kleinhirns hinzog; 2. eine ebenfalls absteigende Degeneration eines grossen, von mir unter der Bezeichnung der centralen Haubenbahn beschriebenen Bündels, welche man nach unten bis zu der entsprechenden unteren Olive verfolgen konnte und 3. eine Degeneration der linken Schieifenschicht, welche von dem erwähnten Herd nach oben bis in den Sehhügel und nach unten bis in die Kerue der Hinterstränge, welche atrophisch waren, hineinragte.

Ueber die Degeneration der Goll'schen Bündel, der Pyramide und der Pyramidenbündel ist es unmöthig, hier sich weiter auszulassen, da die Degenerationen dieser Gebilde sich durch die auf ihrer Bahn localisirten Herde erklären lassen, ihre Lage eine gewöhnliche war und sie überhaupt nichts weiter Beachtenswerthes darboten. Im Gegentheil verdient die Degeneration der Fasern des vorderen Kleinhirnschenkels und des centralen Haubenbündels unsererseits volle Beachtung, da sowohl jene wie auch diese bis auf die Gegenwart noch lange nicht genügend erforscht ist.

Soviel mir bekannt ist, existirt in der Frage über die secundäre, absteigende Degeneration der Fasern des vorderen Kleinhirnschenkels in der Literatur unter Anderem eine Beobachtung von Prof. E. Mendel*). In seinem Falle handelte es sich um eine Affection des einen Sehhügels, welche eine secundäre absteigende Degeneration eines unter dem Vierhügel sich kreuzenden und alsdann zu der contralateralen Hälfte des Kleinhirns gehenden Bündels im vorderen Kleinhirnschenkel zur Folge hatte. In unserem Falle befand sich der sklerotische Herd unter dem hinteren Zweihügel, hatte somit die Fasern des vorderen Kleinhirnschenkels schon hinter ihrer allgemeinen Kretzungsstelle ergriffen. Hieraus wird es verständlich, warum das degenerirte Bündel in unserem Falle in dem entsprechenden Kleinhịnschenkel sịch befin-

*) Mendel, Neurol. Centralbl. 1885. 
den musste. Jedenfalls ist es mehr als wahrscheinlich, dass in unserem Falle dasselbe Bündel wie in dem Mendel'schen Falle degenerirt war.

$\mathrm{Zu}$ bemerken ist noch, dass die neuesten, nach der Golgi'schen Methode ausgeführten Untersuchungen uns davon überzeugten, dass die vorderen Kleinhirnschenkel nicht allein aus den von den centralen Kleinhirnzellen ausgehenden Fasern bestehen, sondern auch aus solchen von entgegengesetzter Richtung, welche aus den Zellen der rothen Kerne hervorgehen. Diese Untersuchungen, in Verbindung mit den oben angeführten Beobachtungen über die secundäre absteigende Degeneration eines Theiles der Fasern des vorderen Kleinhirnschenkels erlauben somit nicht mehr daran zu zweifeln, dass im letzteren ausser den centripetalen, zum Grosshirn aufsteigenden Fasern noch ein centrifugales Fasersystem enthalten ist.

Was die absteigende Degeneration des von mir beschriebenen centralen Haubenbündels anbetrifft, so ist sie schon wiederholt beobachtet worden. An Präparaten von einem Anencephalos, welche Professor R. Flechsig die Güte hatte, mir 1885 zu zeigen, war dieses Bündel an beiden Seiten vollständig degenerirt vorgefunden worden, wobei auch die unteren Oliven atrophisch erschienen. Ebenso findet man nicht selten in den Fällen von bedeutenden Zerstörungen der Hemisphären bei Idioten eine Atrophie des centralen Haubenbündels, zusammen mit einer solchen der entsprechenden unteren Olive ${ }^{*}$ ).

In einem Falle von Taubstummheit hatte ich auch Grelegenheit eine einseitige, absteigende Degeneration des Haubenbündels zu beobachten.

Somit kann gegenwärtig, in Anbstracht aller soeben angeführten Beobachtungen, es als unzweifelhaft gelten, dass das centrale Haubenbündel in pathologischen, zur Unterbrechung seiner Fasern führenden Fällen in absteigender Richtung degenerirt, folglich ein centrifugal verlaufendes Fasersystem repräsentirt.

In unserem Falle verdient endlich noch die auf- und absteigende Degeneration der Schleifenschicht Beachtung. In aufsteigender Richtung reichte die Degeneration der Schleifenschicht nur bis zu den Sehhügeln, welcher Umstand augenscheinlich für eine wenigstens theilweise Endigung der Schleifenschicht im Sehhügel spricht, wovon ich mich auch durch Untersuchungen, welche nach der entwickelungsgeschichtlichen Methode ansgeführt worden waren, überzeugt habe ${ }^{* *}$ ). Was die absteigende Degeneration anbelangt, so war dieselbe in unserem Falle durch

*) Vergl. Legersma, Schmidt's Jahrb. Bd. CCXIX.

**) Siehe meine Arbeit "Ueber die Schleifenschicht" im Neurol. Centralbl. 1885. (Mittheilung von P. Flechsig) und im Neurol. Centralbl. Lief. 3. 1895. 
die Olivenzwischenschicht und die hintere Kreuzung bis zu den deutlich atrophisch erscheinenden Hinterstrangkernen der entgegengesetzten Seite zu verfolgen. Ohne auf die Einzelheiten bezüglich dieser Degeneration näher einzugehen, wollen wir hier nur erwähnen, dass in der Literatur schon wiederholt Fälle von auf- und absteigender Schleifendegeneration veröffentlicht worden sind; einige Uale aber ist die Degeneration der Schleifenschicht, gleich unserem Falle, gleichzeitig sowohl in auf- wie absteigender Richtung vorgefunden worden. Solche Daten sprechen augenscheinlich dafür, dass in der Schleifenschicht sowohl auf- wie absteigende Fasersysteme enthalten sind, diese Schicht folglich Fasern von verschiedener Bedeutung aufweist.

\section{Erklärung der Abbildungen (Taf, XVII.).}

Fig. 1. Der Schnitt geht durch die Auftreibung der Kleinhimarterie; innerhalb des Gefässes sieht man die Organisation des Thrombus. .

Fig. 2. Ein Schnitt neben der aufgetriebenen Stelle der Kleinhirnarterie. Die Abbildung zeigt eine auffallende Verdickung der Gefässwandung mit reichlicher Entwickelung der Granulationselemente, besonders zwischen der Adventitia und der Media. Rechts sieht man einen Theil der von dem Schnitt mitbetroffenen Verdickung mit dem sich organirenden Thrombus in derselben; links ein obliterirtes kleines Gefäss.

Fig. 3. Vorderer Theil eines Querschnittes vom Brustmark mit zwei Herden der syphilitischen Sklerose. In der weissen vom Herd verschonten Rückenmarkssubstanz erkennt man eine Degeneration beider vorderen und des linken seitlichen Pyramidenbündels, eine Degeneration des Vorderaussenbündels Iinks und eine theilweise Degeneration der Fasern an der Grenze zwischen dem Vorder- und Seitenstrange der linken Seite.

Fig. 4. Ein Herd der syphilitischen Sklerose aus der Gegend des linken Corpus restiforme. Links die äussere Oberfläche des von dem Herd betroffenen Corpus restiforme.

Fig. 5. Querschnitt im Niveau der Hirnschenkel unter dem vorderen Zweihügel, über dem in der linken Haubenhälfte localisirten Herd der Sklerose. Man erblickt eine aufsteigende Degeneration der linken Schleifenschicht und eine Atrophie der Fasern des vorderen Kleinhirnschenkels rechts, oberhalb der Kreuzung. In der unteren Etage bemerkt man im medialen Hirnschenkeltheil eine Degeneration der Fasern. Gefärbt nach Weigert.

Fig. 6. Schnitt aus dem Nivean des hinteren Hirnschenkelabschnittes, unmittelbar oberhalb des sklerotischen Herdes, in derGegend der linken Haubenhälfte. Aufsteigende Degeneration der Fasern der Schleife; fast vollhommene Abwesenheit des centralen Haubenbündels links; Atrophie des linken Kleinhirnschenleels und Degeneration des inneren Ahschnittes des linken Hirnschenkels. Gefärbt nach Weigert. 
772 Prof. W. v. Bechterew, Ueber syph. dissem., cerebrosp. Sklerose.

Fig. 7. Querschnitt im Niveau des hinteren Zweihügels. Bei S. sklerotischer, sich auf einen Theil des vorderen Kleinhirnschenkels, des centralen Haubenbündels und der Schleifenschicht erstreckenden Herdes. In der Brücke links bei a eine Hämorrhagie. Deutlich ausgesprochene Degeneration des Pyramidenbündels. Tinction nach Weigert.

Fig. 8. Querschnitt im Niveau des mittleren Brückentheiles. Am Präparate erkennt man in der unteren Etage eine Degeneration der Pyramiden, in der Haube eine Degeneration eines der Bündel des vorderen Kleinhirnschenkels, des centralen Haubenbündels links und der Schleifenschicht. Tinction nach Weigert.

Fig. 9. Querschnitt im Niveau des unteren Brückentheils. Sklerose in der Gegend der linken Schleifenschicht und in der linken Brückenhälfte; bei a eine Hämorrhagie. Tinction nach Weigert.

Fig. 10. Querschnitt im Niveau des unteren Theiles der Brücke. (Die rechte Hälfte der Abbildung entspricht der linken Hirnhälfte.) Wan sieht eine Degeneration der Schleifenschift und des centralen Haubenbündels. Die Pyramiden sind vom Präparate entfernt. Tinction nach Weigert.

Fig. 11. Schnitt im Niveau des verlängerten Markes. Rechts: Atrophie der Hinterstrangkerne und der Formatio reticularis. Tinction nach Weigert.

Fig. 12. Vordere Rückenmarkfläche; die Stellen, an welchen sich die sklerotischen Herde befanden, sind schraffirt. 
.
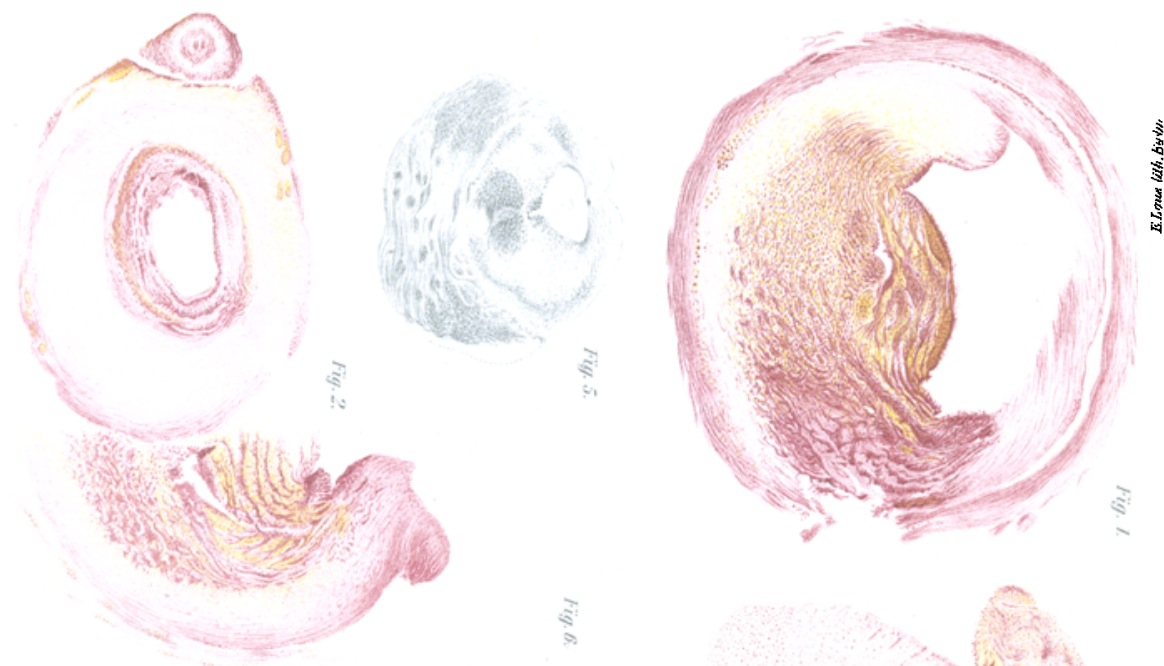

:
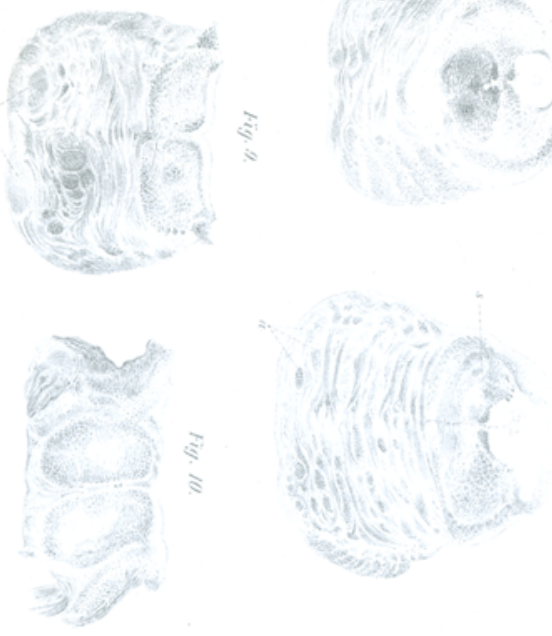

है

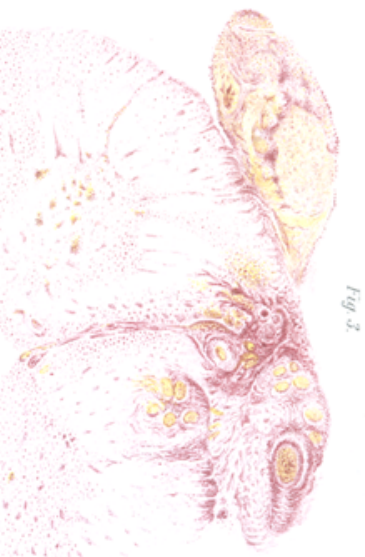

1) $\mathrm{x}$
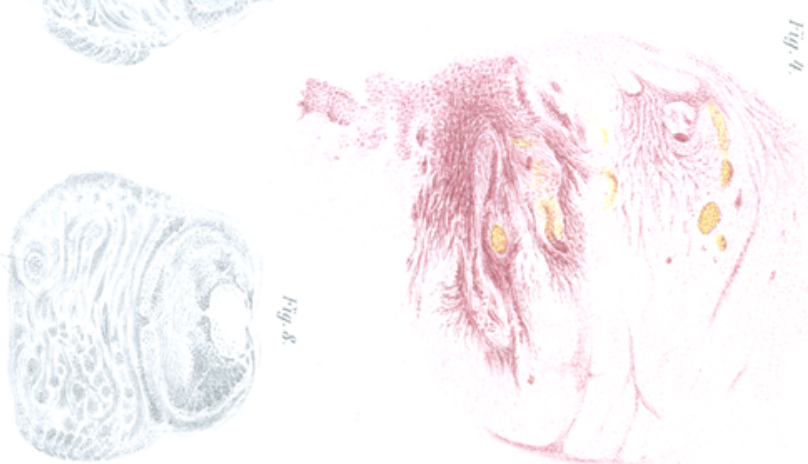
s
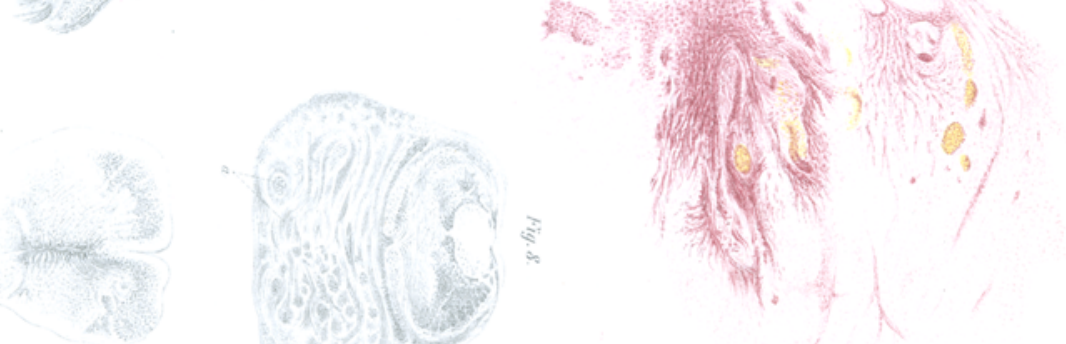

ป

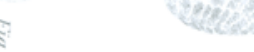

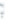

.

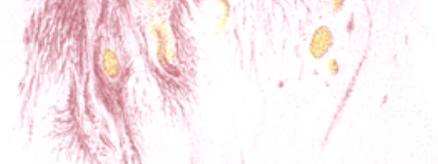

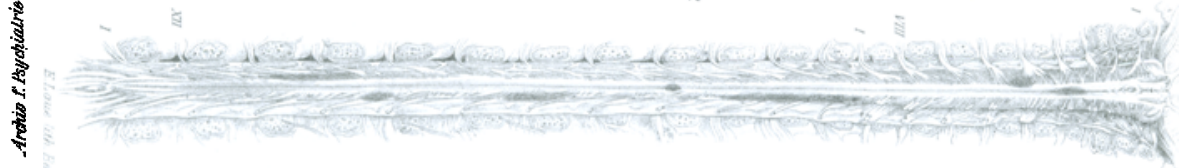

